Cahiers $d u$ MONDE RUSSE

\section{Cahiers du monde russe}

Russie - Empire russe - Union soviétique et États indépendants

49/2-3 | 2008

Sortie de guerre

\title{
Expérience de guerre, hiérarchie des victimes et justice sociale à la soviétique
}

\author{
Nathalie Moine
}

\section{OpenEdition}

Journals

Édition électronique

URL : https://journals.openedition.org/monderusse/9136

DOI : 10.4000/monderusse.9136

ISSN : $1777-5388$

Éditeur

Éditions de l'EHESS

Édition imprimée

Date de publication : 20 septembre 2008

Pagination : 383-418

ISBN : 978-2-7132-2196-5

ISSN : $1252-6576$

Référence électronique

Nathalie Moine, "Expérience de guerre, hiérarchie des victimes et justice sociale à la soviétique », Cahiers du monde russe [En ligne], 49/2-3 | 2008, mis en ligne le 01 janvier 2011, consulté le 04 septembre 2022. URL : http://journals.openedition.org/monderusse/9136 ; DOI : https://doi.org/ 10.4000/monderusse. 9136 
chercher : repérer : avancer

Cet article est disponible en ligne à l'adresse :

http://www.cairn.info/article.php?ID REVUE=CMR\&ID NUMPUBLIE=CMR 49\&ID ARTICLE=CMR 4920383

\title{
Expérience de guerre, hiérarchie des victimes et justice sociale à la soviétique
}

\author{
par Nathalie MOINE
}

\section{Editions de l'EHESS | Cahiers du monde russe}

\section{8/2-3 - Vol 49}

ISSN 1252-6576 | ISBN 9782713221965 | pages 383 à 418

\section{Pour citer cet article :}

- Moine N., Expérience de guerre, hiérarchie des victimes et justice sociale à la soviétique, Cahiers du monde russe 2008/2-3, Vol 49, p. 383-418.

Distribution électronique Cairn pour les Editions de l'EHESS.

(C) Editions de l'EHESS. Tous droits réservés pour tous pays.

La reproduction ou représentation de cet article, notamment par photocopie, n'est autorisée que dans les limites des conditions générales d'utilisation du site ou, le cas échéant, des conditions générales de la licence souscrite par votre établissement. Toute autre reproduction ou représentation, en tout ou partie, sous quelque forme et de quelque manière que ce soit, est interdite sauf accord préalable et écrit de l'éditeur, en dehors des cas prévus par la législation en vigueur en France. Il est précisé que son stockage dans une base de données est également interdit. 


\section{EXPÉRIENCE DE GUERRE, HIÉRARCHIE DES VICTIMES ET JUSTICE SOCIALE À LA SOVIÉTIQUE}

Malgré l'abondance des publications sur l'histoire de la Seconde Guerre mondiale
en Union Soviétique, un certain nombre d'aspects concernant l'expérience des
populations civiles dans les territoires occupés, particulièrement dans la partie
appelée par les Allemands « la vieille Russie », demeurent obscurs ${ }^{1}$. De la même

1. Les travaux les plus fouillés sur l'expérience d'occupation par les civils soviétiques, grâce à l'accès relativement récent aux archives soviétiques, concernent avant tout la Biélorussie (Christian Gerlach, Kalkulierte Morde: Die deutsche Wirtschafts- und Vernichtungspolitik in Weissrussland, Hamburg : Hamburger Edition, 1999 ; Bernhard Chiari, Alltag hinter der Front: Besatzung, Kollaboration und Widerstand in Weissrussland, 1941-1944, Düsseldorf : Drofte, 1999) ainsi que l'Ukraine (Karel Berkhoff C., Harvest of Despair: Life and Death in Ukraine under Nazi Rule, Cambridge, MA : Belknap Press of Harvard University Press, 2004 ; Tanja Penter, « Die lokale Gesellschaft im Donbass unter deutscher Okkupation 1941-1943 », Beiträge zur Geschichte des Nationalsozialismus, 19, 2003, p. 183-223 et « Zwangsarbeit-Arbeit für den Feind. Der Donbass unter deutscher Okkupation (1941-1943) », Geschichte und Gesellschaft, 31, 2005, p. 68-100 ; Amir Weiner, Making Sense of the War: The Second World War and the Bolshevik Revolution, Princeton : Princeton Univ. Press, 2001). En ce qui concerne la Russie soviétique, des problèmes de documentation (sous administration militaire dont beaucoup d'archives furent détruites) et des problèmes de positionnement historiographique (le renouvellement des travaux sur l'occupation est venu pour beaucoup des recherches consacrées à l'histoire de la Shoah en territoire soviétique, c'est-à-dire avant tout dans les territoires occidentaux de l'URSS qui comptaient les plus fortes concentrations de populations juives au moment de l'invasion) expliquent le moindre avancement des connaissances sur la période. Il faut signaler cependant, la notable exception constituée par le travail très riche et très novateur de B.H. Kovalev, Nacistskij okkupacionnyj režim i kollaboracionizm v Rossii (1941-1944 gg.) [Le régime d'occupation nazi et la collaboration en Russie, 1941-1944], Velikij Novgorod : Novgorodskij gos. Universitet, 2001. Les travaux de Dallin, Mulligan et Brandt à partir des sources allemandes restent donc essentiels pour la partie russe de l'URSS occupée : Alexander Dallin, German rule in Russia 1941-1945: a study of occupation politics, London: Macmillan, 1986 ; Karl Brandt, Management of Agriculture and Food in the German-occupied and other areas of Fortress Europe: a Study in Military Government, Stanford : Stanford University Press, 1953 ; Timothy Patrick Mulligan, The Politics of Illusion and Empire : German Occupation Policy in the Soviet Union, 1942-1943, New York : Praeger, 1988. Cf. aussi la remarquable synthèse proposée par Dieter Pohl, Die Herrschaft der Wehrmacht: Deutsche Militärbesatzung und einheimische Bevölkerung in der Sowjetunion 1941-1944, Munich : Oldenbourg, 2008. 
façon, la sortie de guerre reste largement une zone d'ombre sur toute une série de questions liées à la reconstruction de la vie des civils. Les souffrances inouïes dont furent victimes les populations civiles en territoire occupé sont bien au premier plan des descriptions publiées dès la guerre par les services d'information et de propagande du gouvernement soviétique. Elles font pleinement mention, outre les violences faites au corps, des destructions et pillages perpétrés à l'encontre des biens propres des civils. Les dégâts matériels subis par les civils sont également dûment répertoriés dans les bilans effectués et publiés à la libération. Cependant, le silence se fit ensuite sur le devenir de ces populations qui avaient pourtant perdu l'essentiel de leurs biens. L'historiographie soviétique se concentra sur la reconstruction de l'appareil de production et des équipements collectifs, mais elle ne dit plus un mot sur la difficile reconstitution par les civils d'un patrimoine privé, ne serait-ce que minimal : reconstruire un toit, se vêtir, se meubler. L'historiographie actuelle a considérablement renouvelé les connaissances sur l'après-guerre soviétique mais, focalisée sur l'analyse des processus de répression, elle a, elle aussi, négligé les aspects matériels de la sortie de guerre, sinon pour souligner la misère générale des populations ${ }^{2}$. Les études sur la famine de 1946 font exception mais elles ne relient pas leur analyse socioéconomique à la question de la sortie d'occupation. De plus, elles portent avant tout sur les problèmes de ravitaillement ${ }^{3}$.

C'est précisément la question du devenir des populations sinistrées après la libération des territoires occupés que nous entendons aborder ici. L'étude des logiques mises en œuvre dans la distribution de l'aide matérielle aux civils renvoie aux modes de catégorisation des victimes de l'occupation, en écho aux études sur l'histoire sociale du stalinisme dans les années 19304. Comprendre l'importance accordée à l'expérience de la guerre par les individus dans ces logiques de distribution constitue la question centrale de cette étude 5 . Le rôle de la guerre dans l'aide sociale se traduit en effet par la place nouvelle occupée dans la hiérarchisation des ayants droit comme les familles de soldats. Dans quelle mesure joua également l'attitude des individus restés en territoire occupé, que celle-ci ait été suspectée de

2. Elena Zubkova, Russia After the War: Hopes, Illusions and Disappointments, 1945-1957, Armonk, New York : M. E. Sharpe, 1998.

3. V.P. Popov, Ekonomičeskaja politika sovetskogo gosudarstva, 1946-1953 [La politique économique de l'État soviétique, 1946-1953], M. : Izd. TGTU, 2000 ; V.F.Zima, Golod v SSSR 1946-1947 godov: proishoždenie i posledstvija [La faim en URSS en 1946-1947: causes et conséquences], M.: Institut rossijskoj istorii RAN, 1996 ; Jean Lévesque, « Part-Time Peasants : Labour Discipline, Collective Farm Life, and the Fate of Socialist Agriculture in the Soviet Union after the Second World War, 1945-1953 », PhD Dissertation, University of Toronto, 2003.

4. Elena Osokina en particulier a bien montré la construction d'une hiérarchie de la pauvreté dans ses travaux sur le ravitaillement. Elena Osokina, Za fasadom «stalinskogo izobilija »: Raspredelenie i rynok v gody industrializacii, 1927-1941 [Derrière la façade de l' « abondance stalinienne » : répartition et marché dans les années de l'industrialisation, 1927-1941], M. : Rosspen, 1998. Voir aussi son article, «Une hiérarchie de la pauvreté. Approvisionnement d'État et stratification sociale en URSS pendant la période de rationnement 1931-1935 », Cahiers du monde russe, 39 (1-2), 1998, p. 81-97.

5. Weiner, Making Sense of War... ; Marc Edele, « Soviet Veterans as an Entitlement Group, 1945-1955 », Slavic Review, 65 (1), 2006, p. 111-137. 
collaboration ou, au contraire reconnue comme contribution à la résistance ? Et dans quelle mesure, pour finir, le niveau de pertes subies par un individu fut-il pris en considération ? Autrement dit, comment se reconfigure la hiérarchie des ayants droit de l'aide sociale soviétique au lendemain de la guerre ? Ce questionnement, qui s'inscrit dans une recherche sur la sortie d'occupation, rend indispensable un retour sur la période de l'occupation elle-même pour mieux analyser ensuite les logiques et les pratiques soviétiques de la sortie de guerre. Moins qu'une évaluation attendue des pertes à travers le lien établi entre les deux séquences - occupation allemande, libération - , rendue très irréaliste en raison des informations trop parcellaires livrées par les archives, l'objectif de cette démarche est, du moins, de contribuer à un état des lieux des connaissances de la guerre vécue par les civils soviétiques, à travers la confrontation des documents conservés des autorités allemandes et soviétiques ${ }^{6}$.

L'approche choisie a été celle d'une échelle micro-historique et concerne un district de la région de Leningrad. Situé à la frontière avec l'Estonie, le district de Slancy est très faiblement peuplé 7 , à la fois en raison des conditions naturelles et de son statut de zone frontalière. La population est majoritairement russe, avec une présence estonienne d'autant plus discrète qu'elle a dû, en grande partie, être évincée pendant l'entre-deux-guerres. L'édification d'un centre industriel, lancée au début du Premier Plan quinquennal à partir d'un gisement de schistes pétrolifères, transforme durablement le profil du district. Bientôt, des baraquements construits à la hâte surgissent autour des mines et usines. Ils accueillent une population jeune, venue pour l'essentiel des villes de la région (notamment Leningrad et Pskov), qui se distingue de la population rurale par un rapport différent au régime, car si elle subit les dures contraintes du travail industriel des grands chantiers construits à marche forcée, elle est aussi davantage exposée à la propagande du régime. L'invasion allemande rend à la région son profil rural, à la suite de l'évacuation du bourg et de la destruction de l'appareil industriel organisées à la hâte par les Soviétiques à l'été 1941. Lorsque l'Armée rouge la libère au début de 1944, elle est à peu près déserte. Une intense activité des mouvements partisans a précédé l'arrivée des soldats de l'Armée rouge et, après les affrontements, le bourg de Slancy n'est plus qu'un tas de ruines, la plupart des villages ont été incendiés. Bien que le district de Slancy se distingue des territoires marqués par des crimes de masse contre les civils que furent l'Ukraine et la Biélorussie, il s'agit néanmoins d'une région totalement sinistrée. Elle a connu son lot quotidien de déportations de masse et d'exécutions sommaires. C'est donc à partir du cas de Slancy que nous allons tenter de restituer

6. Cette démarche de confrontation des sources allemandes et soviétiques sur une échelle locale caractérise notamment le travail pionnier de Tanja Penter. J'ai, pour ma part, bénéficié de la participation précieuse de Gàbor Rittersporn, sans lequel cette recherche n'aurait pas été possible et je lui en suis profondément reconnaissante. Je remercie également pour leur aide et leurs encouragements Alain Blum, Michel Duflo, Catherine Gousseff et Anna Zaytseva.

7. À vrai dire, il compte une population de taille beaucoup plus réduite qu'un district moyen et, de ce fait, plus facile à identifier, tout en correspondant à un maillon administratif permettant un niveau de documentation minimale. 
la « sortie de guerre ». Alors que le terme de « reconstruction» tend à donner pour accompli ce qui n'est qu'un objectif, il faudra tenter de qualifier ce qui se passe dans ce microcosme dépourvu de toutes ressources et dépendant d'un centre, Leningrad, lui-même traumatisé par un long siège de deux ans et demi.

La question des sources apparaît évidemment comme fondamentale dans ce travail de reconstitution à la fois de la guerre et de la sortie de guerre. Pour ces deux périodes, la collecte s'est révélée problématique : en ce qui concerne la guerre, nous avons pu croiser des sources allemandes en provenance à la fois des institutions en charge de l'exploitation économique des territoires occupés et de celles en charge de son contrôle ${ }^{8}$ et, du côté soviétique, les témoignages recueillis par la Commission d'enquête soviétique sur les crimes de guerre nazis ( $\check{C} G K^{9}{ }^{9}$, ainsi que quelques archives en provenance des partisans ${ }^{10}$. En ce qui concerne l'après-guerre, nous nous sommes essentiellement appuyée sur les archives du comité exécutif du soviet du district, l'ispolkom ${ }^{11}$, celles du comité du parti, tant au niveau du district que celui de l'oblast de Leningrad étant largement censurées, sans parler des archives des organes de sécurité. L'aide sociale relevant avant tout de l'administration du soviet local, les archives disponibles permettent de dresser un panorama assez complet des mesures prises, dans lesquelles l'importance des critères politiques transparaît clairement sans pouvoir toutefois être complètement explicitée.

Nous commencerons donc par retracer non pas l'histoire du district sous occupation mais ce qui apparaît comme pertinent vis-à-vis du questionnement autour des modes de redistribution d'après-guerre, à savoir l'attitude des populations visà-vis tant de l'occupant que du mouvement partisan et les circonstances des destructions. Nous retracerons ensuite la restauration du pouvoir soviétique dans les campagnes du district ainsi que la reconstitution des formes d'aide aux populations sinistrées. Il s'agira ici de s'interroger sur la mise en œuvre d'une justice sociale « à la soviétique », combinant aide aux catégories définies par le pouvoir central et aide aux plus miséreux repérés par les pouvoirs locaux.

8. Archives du commando économique en charge du district de Gdov dans lequel s'inscrit pendant la guerre celui de Slancy, BA-MA (BundesArkhiv-Militär Arkhiv, Freiburg), RW31 (WirtschaftsstabOst), dossiers 927-933 ; archives de la division de sécurité couvrant un vaste territoire à cheval sur l'Estonie et la Russie du Nord Ouest, la division 207 (BA-MA, RH 26207 (Sicherheit-Division 207, Rückw. Heeresgebiet Nord). Sur les structures locales de l'administration militaire d'occupation, cf. Pohl, Die Herrschaft der Wehrmacht...

9. GARF (Gosudarstvennyj arhiv Rossijskoj Federacii - Archives d'État de la Fédération de Russie), f. 7021, ČGK, op. 30 (oblast de Leningrad). Cette commission a été créée par les Soviétiques à la fin 1942 pour enquêter sur les crimes de guerre commis par l'occupant et commence son gigantesque travail d'inventaire à partir du printemps 1943, au fur et à mesure de la libération des territoires occupés. Pour une présentation générale du travail des commissions locales de la ČGK, cf. Nathalie Moine, «La Commission d'enquête soviétique sur les crimes de guerre nazis : entre reconquête du territoire, écriture du récit de la guerre et usages justiciers », Le Mouvement Social, 222, janvier-mars 2008, p. 81-109.

10. Archives pour l'essentiel conservées dans le musée de la ville de Slancy (Slancevskij Istoričeskij Kraevedčeskij Muzej).

11. LOGAV (Leningradskij oblastnoj gosudarstvennyj arhiv v g. Vyborge - Archives d'État de l'oblast de Leningrad dans la ville de Vyborg), f. 624. 


\section{Slancy sous l'occupation}

La population de sinistrés dont il est question ici est constituée essentiellement des ruraux, ceux qui sont restés sur place pendant l'occupation. Quelques mots sur le bourg lui-même paraissent cependant indispensables puisque, tant sous les Soviétiques que pendant l'occupation allemande, Slancy constitue pour les ruraux un pôle, étranger mais incontournable, où se trouve concentré le pouvoir administratif de la puissance du moment, et qui se présente aussi comme un lieu d'embauche en dehors des travaux agricoles. Le bourg de Slancy, qui n'est encore qu'un grand chantier au moment de l'invasion allemande, a bien failli être définitivement rayé de la carte par la guerre. L'évacuation a lieu au début du mois de juillet 1941, alors que les hommes du bourg sont appelés sous les drapeaux ou rejoignent les premières formations de partisans. Les installations qui ne sont pas évacuées sont détruites. Le bourg de Slancy tombe entre les mains des Allemands dans la nuit du 17 au 18 juillet 1941, c'est-à-dire dans un trou noir du point de vue des sources soviétiques. De façon symptomatique, la Commission extraordinaire d'enquête sur les crimes de guerre allemands a archivé des photos prises de Slancy en ruines, au moment de sa libération, sans qu'on sache à quoi ressemblait le bourg sous l'occupation. De fait, les Allemands ont, tout comme les Soviétiques en 1941, rendu les équipements industriels impraticables, par une politique systématique de démontage et de destruction, au moment de leur retraite. Cependant, entre le départ des Soviétiques et celui des Allemands s'étirent plus de deux années au cours desquelles le bourg retrouve progressivement une activité industrielle et administrative. Comme nous le verrons plus loin, les témoignages recueillis par la Commission extraordinaire d'enquête présentent la ville comme le principal lieu de la terreur, et non comme un centre administratif et industriel. En taisant ces derniers aspects et en réduisant Slancy à l'épicentre de la terreur, les Soviétiques évincèrent ainsi les traces de la participation des locaux, forcée ou volontaire, au quotidien de l'occupation, à des niveaux divers de spécialisation et d'implication. Les sources allemandes montrent en particulier les tentatives faites pour remettre en activité les installations de Slancy, afin d'inscrire sa production au sein du bassin plus large de la Baltique. Cet objectif impliquait pour les autorités allemandes en poste dans la région de résoudre le problème de la main-d'œuvre. En effet, les besoins étaient loin d'être anecdotiques compte tenu du dépeuplement croissant de la région et bien qu'elle ait connu un afflux considérable de population réfugiée en provenance des alentours de Leningrad, déplacée plus à l'Ouest par les Allemands ${ }^{12}$. La ponction du printemps 1942 pour envoyer des travailleurs vers le Reich a produit son effet. En juillet 1942, le département Travail du commando économique (Wirtschaftskommando), basé à Gdov, ouvre une annexe à Slancy afin de mieux suivre les exigences en maind'œuvre des établissements industriels ${ }^{13}$. À la fin septembre, le commando note avec satisfaction que cent personnes supplémentaires ont été trouvées pour le travail de

12. BA-MA, RH26-207/13, 15000 réfugiés passés dans la région de Gdow au 13.02.42.

13. BA-MA, RW31/928, entrée du 15.07.42. 
sauvegarde du bois. Cependant, les plus gros besoins viennent des activités prises en main par la Baltische Oil GmbH à Slancy : ceux-ci doivent être couverts par des gens de l'extérieur, car il n'y a presque plus de civils valides disponibles dans l'ensemble du territoire couvert par le commando. La présence de Frontarbeiter hollandais, venus dans le cadre du Service du travail, est ainsi mentionnée et a permis de combler une partie des besoins en travailleurs qualifiés. En ce qui concerne le travail non qualifié, les autorités allemandes ont également recours aux prisonniers de guerre soviétiques, qui apparaissent parfois dans les témoignages des habitants recueillis au sortir de la guerre par les enquêteurs soviétiques, évoquant leur travail dans les entreprises de Slancy à leurs côtés. Certains, soupçonnés de vouloir faire exploser la fabrique dans laquelle ils travaillaient, sont exécutés dans la pinède de Gavrilovskoe ${ }^{14}$. Le commando économique allemand mentionne en décembre 1942 que l'attribution de 2000 prisonniers de guerre soviétiques a soulagé la question de la main-d'œuvre à Slancy. Cependant, cet apport est des plus fragiles: à la fin décembre, l'examen médical des 401 hommes du SonderStrafbatalion arrivés pour travailler dans les Brennschifswerke de Slancy conclut à leur incapacité physique, à l'exception de 82 d'entre eux au maximum ${ }^{15}$.

Slancy est donc le lieu d'installation des occupants, comprenant plusieurs bureaux dans lesquels travaillent des Allemands mais aussi des auxiliaires, Estoniens et Russes. C'est le principal lieu de contact entre les locaux et les occupants, en dehors des descentes dans les villages.

S'il est le point de contact le plus proche, il n'est pas le seul pour autant : des localités plus importantes comme Gdov, Kingissepp ne sont qu'à quelques dizaines de kilomètres, mais ces localités paraissent encore plus lointaines dans les témoignages que Slancy, et leur absence reflète la mobilité extrêmement réduite de la population dans les territoires occupés.

\section{L'occupation dans les zones rurales du district}

Si l'on s'en tient aux récits officiels, il n'est guère plus facile de se représenter le temps de l'occupation dans les zones rurales du district. Cependant, la paysannerie se fait entendre dans les témoignages recueillis au cours de l'immédiat après-guerre (et conservés dans les archives de la Commission extraordinaire). Ils valent la peine de s'y attarder, même s'ils ne couvrent pas tous les aspects de l'occupation, notamment les structures administratives et l'organisation économique. Les témoignages des ruraux après-guerre taisent ainsi toute transaction avec les Allemands pour se concentrer sur les actes de terreur.

Les échanges économiques au quotidien entre occupants et occupés constituent un aspect de l'occupation demeuré encore très mal connu, en particulier pour la Russie, si ce n'est la conclusion fondamentale sur l'exploitation meurtrière opérée

14. GARF, f. 7021, op. 30, d. 250a, 1. 196 ob

15. BA-MA, RW31/928, entrées du 30.09.42, 1.12.42 et 22.12.42. 
par les nazis. Il transparaît cependant du côté allemand ${ }^{16}$. Les journaux de service (Tagebücher) du Wirtschaftskommando de la région de Gdov rapportent les progrès des récoltes et semblent ne pas trop se plaindre de l'attitude de la paysannerie :

Le dimanche 8 mars [1942], devant l'église de Gdov le responsable du commando économique, le major Rusche a annoncé à la population l'entrée en vigueur du nouveau système agraire. Étaient présents tous les officiers et employés civils du cantonnement de Gdov. Il semble que ce système ait été, comme ailleurs, favorablement accueilli par la population de cette région économique. D'après l'expérience des jours suivants, les paysans font montre d'un plus grand empressement à donner suite aux ordres donnés par les bureaux militaires et économiques, ${ }^{17}$

écrit le capitaine Von Hagen à l'entrée du 15 mars 1942 du journal d'activité du commando. En revanche, pas trace, dans les témoignages soviétiques d'aprèsguerre, de ce nouvel ordre agraire, édicté le 16 février 1942 et objet d'une propagande intense par les autorités d'occupation, et encore moins d'une quelconque satisfaction face aux nouvelles opportunités offertes par les occupants démantelant le système honni des kolkhozes. Cependant, la nécessité pour les autorités soviétiques à la Libération de remettre de l'ordre dans les kolkhozes en redéfinissant les statuts de propriété indique bien des modifications profondes opérées sous l'occupation. Dans ce district, comme pour l'ensemble de la région, il n'est guère possible de mesurer avec précision la réalité et l'ampleur de la réorganisation agraire. Si nous pouvons supposer qu'elle fut, comme dans le reste de la région, essentiellement de papier, les kolkhozes devenant des sortes de coopératives agricoles (Landbaugenossenschaft), il n'empêche que le système fut alors ébranlé. Dès la période de vacance de pouvoir entre le départ précipité des autorités soviétiques et l'installation des autorités allemandes, la dissolution spontanée de certains kolkhozes est avérée. Ailleurs, on se répartit tout ou partie des biens du kolkhoze et de ses terres. Plus tard, certains cultivateurs obtinrent d'agrandir leurs lopins de ménage (priusadebnoe hozjaistvo) en échange de leur coopération active avec l'occupant ${ }^{18}$. De toute façon, à défaut de véritablement modifier les structures de propriété, les autorités allemandes avaient autorisé, à partir de l'été 1941, 1'assouplissement des règles drastiques en matière de taille des lopins (Hofland) et de possession de bétail ${ }^{19}$. Deux Landwirtschaftssonderführer, Nötzelmann et Kern, officient pour le poste de Slancy à partir de mai $1942^{20}$.

16. On dispose depuis longtemps de descriptions générales sur le système d'exploitation économique des territoires de l'Est. Ces études restent cependant très elliptiques en ce qui concerne la Russie du Nord-Ouest, sous administration militaire. Cf. Dallin, German rule... ; Mulligan, The Politics of Illusion... ; Brandt, Management of Agriculture... ; Pohl, Die Herrschaft der Wehrmacht... et surtout Kovalev, Nacistskij okkupacionnyj režim...

17. BA-MA RW31/927, entrée du 15.03.42.

18. Alexander Hill, The War in the Eastern Front: The Soviet Partisan Movement in NorthWest Russia, 1941-1944, New York : Frank Cass, 2005, p. 57, 101, 105.

19. Gerlach, Kalkulierte Morde..., p. 344, cite Dallin, $334 \mathrm{ff}$.

20. RW31/928, entrée 15.05.42. 
Alors que les employés allemands sont absents des récits, le maillon concret de médiation entre les paysans et les occupants est constitué par les starostes, qui représentent le dernier échelon de l'échelle de l'administration d'occupation locale. La seule interaction décrite dans les témoignages d'après-guerre entre occupants, starostes et villageois correspond aux circonstances dramatiques de l'envoi de maind'œuvre en Allemagne, en particulier lors des opérations du printemps 1942². À la fin mars 1942, une commission de trois personnes, dépêchée par le ministère du Travail (Reichsarbeitministerium) dans le district de Gdov, est chargée de recruter 2400 locaux, hommes et femmes, pour travailler dans l'agriculture allemande. Le département travail du commando économique y met ses meilleures compétences pour les assister ${ }^{22}$. Plusieurs convois quittent donc la région de Gdov avec leur chargement d'Ostarbeiter au cours du mois d'avril23. Cependant, les témoignages des villageois, qui se trouvent être parfois les propres parents des jeunes déportés, évoquent un recrutement et des départs un peu plus tardifs, s'échelonnant entre mai et juin 1942, dans les villages du district de Slancy. D'après les témoignages, quelques Allemands arrivaient dans le village, ordonnaient à la population de se rassembler, sélectionnaient certains villageois en leur prenant leurs papiers d'identité. Les personnes désignées recevaient alors l'ordre de se rendre à la Kommandantur de Slancy, où elles étaient examinées par une commission et envoyées de force, dans un train de marchandise, vers l'Allemagne. Dans plusieurs témoignages, le staroste du village joue, sur ordre des Allemands, un rôle actif dans la désignation des victimes. Les témoins ne donnent jamais la destination précise des Ostarbeiter. Les jeunes gens ont été envoyés en Allemagne et, en 1944, les témoins sont toujours sans nouvelles des déportés.

Cependant, les témoignages recueillis auprès des habitants dressent un tableau complexe, dans lequel l'occupant est loin d'être le seul fléau. Au contraire, la participation de certains habitants du district à la terreur nazie est à plusieurs reprises évoquée. La figure même de l'occupant reste relativement floue. Pas de nom de famille, de numéros d'unité, de type de troupes. En revanche, aux côtés des Allemands, sont mentionnés à plusieurs reprises des Estoniens, qui ne paraissent leur céder en rien en cruauté. Alors que dans d'autres régions soumises à des occupants non allemands (Hongrois, etc.), les habitants n'établissent pas de distinction entre soldats des différentes puissances occupantes, la proximité du district de Slancy avec l'Estonie rend ces « voisins collaborateurs » facilement identifiables ${ }^{24}$.

21. Pohl, Die Herrschaft der Wehrmacht..., p. 310-315, pour l'ensemble des territoires occupés. Pavel Polian, Žertvy dvuh diktatur [Les victimes de deux dictatures], M. : Rosspen, 2002.

22. RW31/927, entrée 31.03.42.

23. 633 personnes envoyées à Ulm dans la première moitié d'avril 1942, 751 le 23 avril, puis 807. Cependant, un autre convoi est également mentionné en mai 1942. BA-MA, RW31/928, entrées $15.04 .42,30.04 .42,31.05 .42$.

24. Sur les Estoniens voir aussi les témoignages recueillis dans le district de Gdov en 2000 et 2003, cités in Žanna Kormina, Sergej Štyrkov, « Nikto ne zabyt, ničto ne zabyto. Istorija okkupacii v ustnyh svidetel'stvah »[Personne n'est oublié, rien n'est oublié. Histoire de l'occupation par les témoins], in Mihail Gabovič, éd., Pamiat' o vojne 60 let spustja. Rossija, Germanija, Evropa [Souvenirs de guerre soixante ans après, Russie, Allemagne, Europe], M. : NLO, 2005, p. $222-240$. 
En réalité, la région est sous la coupe de la Sicherheit-Division 207, basée à Dorpat, qui couvrait un vaste espace allant de l'Ouest de l'Estonie à la bordure Nord-Ouest de la région de Leningrad. Pour remédier au manque chronique en hommes, la division a recours à plusieurs types de troupes supplétives : d'une part des Estoniens ; d'autre part des prisonniers de guerre soviétiques recrutés dans les camps de prisonniers en Estonie. Sans grand succès, les autorités allemandes tentent également de mettre sur pied des unités de locaux (Einwohnerkampfabteilungen). Les recrutements commencent au début de 1942: 15 hommes sont recrutés à Slancy à la fin mars, ils sont déjà 41 en avril25.

Les témoignages sont beaucoup plus précis pour identifier les locaux qui ont collaboré à la terreur nazie. Les dénonciations constituent le type de collaboration le plus souvent évoqué et semblent jouer un rôle non négligeable dans le ciblage des victimes. Pendant toute la durée de l'occupation, la répression nazie est placée sous le signe de la lutte contre les partisans, même si ces termes recouvrent visiblement des réalités variées. Dans les tout premiers temps de l'occupation, il semble que ce soit avant tout une façon de désigner la chasse aux anciens cadres soviétiques restés sur place et débusqués à l'aide de voisins malveillants ${ }^{26}$.

Ainsi ce témoignage d'un vieux paysan, Jakov Ivanovič Kuprjanov, recueilli le 26 septembre 1944 :

le $1{ }^{\text {er }}$ septembre 1941, moi et d'autres personnes travaillions dans les champs, les Estoniens sont arrivés, ils nous ont chassés vers le village (village de Lučki, s/svo de Pojkovskij-Gorskij) et nous ont alignés le long du mur de la maison de Mahov. Un officier se tenait devant nous et à ce moment ils ont amené la citoyenne de notre village Smirnova Natal'ja Semenovna, née en 1892, qui a travaillé trois ans comme présidente du kolkhoze avant la guerre et ils ont amené aussi le citoyen Zabaluev Fëdor, ancien ouvrier à l'administration de la mine. Ils les ont attachés à la palissade. L'officier qui se tenait devant les gens [...] a annoncé que Smirnova et Zabaluev allaient être fusillés pour lien actif avec les partisans. Ensuite il a donné l'ordre à un groupe d'une dizaine d'hommes de tirer, et ils ont tiré une première salve, ensuite ce groupe s'est éloigné et un second groupe de cinq hommes a tiré une seconde salve. Ensuite l'officier a donné l'ordre de mettre le feu à deux maisons. Après l'exécution, les cadavres de Smirnova et Zabaluev ont dû rester deux jours près de la palissade. Smirnova et Zabaluev ont été dénoncés pour liens avec les partisans par un ancien ouvrier de l'administration de la mine, dont je ne connais pas le nom de famille, son prénom c'est Volodia, il est passé aux Allemands, et il était aussi présent pendant l'exécution. ${ }^{27}$

25. BA-MA, RH 26-207/13. Apparemment, des membres de la ROA, évoqués par les sources allemandes pour des districts plus au Sud, sont également présents à Slancy. La garnison de Slancy aurait même abrité un centre de formation pour les futurs combattants de l'armée de Vlasov, accueillant jusqu'à 200 «volontaires » en juin 1943. Cf. archives du musée de Slancy, Spravka o položenii v Slancevskom rajone v periode okkupacii nemecko-fašistskimi vojskami [Information sur la situation dans le rajon de Slancy pendant l'occupation par les troupes fascistes allemandes].

26. Hill décrit également une violence à son paroxysme à la fin de l'année 1941 puis durant l'automne-hiver 1943-1944. Hill, The War in the Eastern Front..., p. 114.

27. GARF, f. 7021, op. 30, d. 250a, 1. 40-40 ob. 
Nous retrouvons ici plusieurs éléments de la violence inaugurale de l'occupation. L'importance en hommes et en moyens déployés pour exécuter dans une mise en scène hâtive deux personnes face à tout un village sert à masquer l'absence d'effectifs, au total, pour tenir la région en cas de soulèvement massif. L'avertissement macabre, souligné par le maintien des cadavres au regard de tous, est redoublé par l'incendie de leurs maisons ${ }^{28}$. Le rôle du dénonciateur est certainement fondamental : les hommes en armes ne peuvent cibler leurs victimes sans l'aide d'insiders. Les motivations de ces derniers restent opaques dans les témoignages, la seule certitude étant leur proximité relative, ici la communauté du lieu de travail, avec les victimes. La même proximité est avérée dans la dénonciation d'Il'ja Stepanovič Burakov par Lidija Aleksandrovna Larenova, qui l'aurait menacé lors d'une assemblée au village de Gorbovo «Si on m'arrête on me libère, si on t'arrête, tu y restes ». Arrêté le 14 avril 1942, Burakov est fusillé pour lien avec les partisans quelques jours plus tard à Slancy. La dénonciation peut être un moyen de se couvrir : soupçonnée d'aider les partisans, une jeune fille dénonce une villageoise qui fabrique du pain pour eux, et finit par quitter la région dans les bagages de l'ennemi ${ }^{29}$. La proximité avec l'occupant n'est pas pour autant un gage d'invulnérabilité et la question de l'appartenance à tel ou tel camp se trouble parfois. Il en va ainsi de Pavel Efimovič Efimov, qui travaille pour les Allemands comme approvisionneur en viande, œufs et autres denrées. Jugeant les livraisons de ce dernier insuffisantes, les Allemands le soupçonnent d'en donner une partie aux partisans, l'arrêtent et le fusillent aux abords de Vejno en juin 1942. Ou encore du meunier Egor Gvozdev, qui dénonce le staroste Ivan Alekseevič Sysoev venu lui apporter le blé qu'il a rassemblé dans son village pour en faire du pain pour les partisans. Cette dénonciation ne semble pas être la seule dans la carrière du meunier, qui «tombe » lui-même en décembre 1942. Accusé d'avoir volé du blé à moudre confié par les villageois, il est à son tour fusillé. Le 2 juillet 1942, deux Estoniens et un Allemand font une descente dans le village de Verbitovo pour arrêter son staroste, Makar Efimovič Efim et son adjoint, Grigorij Ivanovič Ivanov. Conduits à Narva, ils sont fusillés quelques jours plus tard, dénoncés tous deux l'un pour avoir un fils chez les partisans, l'autre parce que sa femme a donné de la nourriture aux partisans. Le fils du staroste est également dénoncé et arrêté ${ }^{30}$. Tous ces cas traduisent le caractère complexe de l'engagement dans un camp ou un autre, que ce soit au niveau des familles ou même d'un individu.

Alors que les autorités allemandes en charge de la sécurité se plaignent du manque d'effectifs, les témoignages des villageois mettent en lumière la toute puissance aveugle de l'occupant: la brutalité de la répression, les exécutions sommaires, l'absence de toute explication aux familles qui n'ont plus de nouvelles des proches arrêtés ou n'apprennent leur sort que par la découverte de leurs cadavres.

28. L'incendie des maisons des personnes exécutées au début de l'occupation est attestée à plusieurs reprises. Cf. GARF, f. 7021, op. 30, d. 250 a, 1. 199, 250 ob, 251 ob.

29. GARF, f. 7021, op. 30, d. 250a, 1. 21 ob.

30. GARF, f. 7021, op. 30, d. 250a, 1. 14-16. 


\section{Les terribles mois de l'automne-hiver 1943}

La violence exercée à l'encontre de la population connaît cependant une escalade très nette lors des derniers mois de l'occupation. L'ordre d'évacuer la région plonge en effet les habitants dans la panique, tandis que les attaques des partisans redoublent d'intensité. Le programme d'évacuation, lancé à la fin septembre 1943, comprend plusieurs volets. Il s'agit tout d'abord d'envoyer en Allemagne le maximum de denrées et de matériel, et de détruire le reste avant qu'il ne tombe aux mains des Soviétiques. En ce qui concerne la population, un projet gigantesque entend conduire les habitants vers les régions baltes ${ }^{31}$. Des convois particuliers sont prévus pour les civils qui ont collaboré avec les Allemands. Les populations finnoises et baltes font également l'objet de convois spécifiques ${ }^{32}$.

L'évacuation de la population suscite des réticences parmi les responsables allemands locaux qui en pressentent les conséquences catastrophiques : la population n'est pas en état de supporter de longues journées de marche alors que l'hiver s'installe et qu'aucune infrastructure n'est prévue pour les haltes de nuit. L'hostilité des populations baltes est également invoquée ${ }^{33}$. Si le commandement ne cède pas dans ses intentions, il est néanmoins obligé de modifier à plusieurs reprises son itinéraire. Celui-ci comprend un long trajet de la «Vieille Russie » vers les régions baltes, l'Estonie puis la Lettonie, avec des points de bivouac non aménagés. Un camp de rassemblement des évacués est installé à Slancy.

Avec d'autres citoyens du district j'ai été déportée pour l'Allemagne ${ }^{34}$ en octobre 1943. Le 28 octobre, 12 Allemands ont fait irruption chez moi. J'étais à la maison avec mon mari Savin Aleksej Afanas’evič né en 1904, ma fille Lidija née en 1928, ma fille Nina née en 1933, mon fils né en 1936, mes filles Vera et Barbara nées en 1943. Ils nous ont donné l'ordre de nous préparer immédiatement car nous étions arrêtés et de les suivre. J'ai demandé qu'ils me laissent avec les enfants mais le chef de cette bande, on l'appelait entre nous «La Barbe » s'est mis à crier sur moi, et m'a ordonné de me taire. Nous étions autorisés à ne prendre pratiquement rien avec nous. Ils ont obligé mon mari à atteler le cheval et nous sommes partis. Ils nous ont emmenés au pont près de Slancy. Ils nous ont retenus là quelque temps, et ensuite ils nous ont entraînés dans un camp dans les cantonnements militaires. Ils nous ont fait passer la nuit dans le camp et le jour suivant ils nous ont chargés dans un convoi et nous ont expédiés vers la Lettonie, où nous avons dû travailler chez des fermiers lettons. Ils ont arrêté mon mari en janvier et l'ont envoyé vers une destination inconnue. Je ne sais rien de ce qui lui est arrivé depuis, car j'étais alitée, malade, au moment de son arrestation. Et ce

31. BA-MA, RH26-207/ 24a, 25b. Cf. aussi Pohl, Die Herrschaft der Wehrmacht..., p. $322-$ 331 .

32. BA-MA, RH26-207/23, p. 77. Les termes utilisés sont Finnen, Ingermanländer, Esten et Letten.

33. BA-MA, RH20-18/1628.

34. Dans ce témoignage comme dans de nombreux autres, la destination donnée, l'Allemagne, sert de terme générique pour désigner un lieu extérieur à la Russie, comme le démontre la suite du témoignage qui précise que les habitants ont été déportés en Lettonie. 
n'est qu'avec l'arrivée de l'Armée rouge que j'ai pu rentrer chez moi avec mes enfants. ${ }^{35}$

Pour échapper à l'évacuation, des villages entiers se cachent dans les forêts, emmenant ce qui reste de bétail et de provisions, que les habitants enfouissent en partie dans le sol. Les Allemands envoient alors la troupe pour tenter de rassembler les villageois, traqués dans la forêt. Les villages sont fouillés, puis incendiés.

Ces actions se déroulent dans un climat de panique croissante, également du côté des Allemands, acculés à la défaite. Alors que les combats se font plus fréquents avec les partisans, les Allemands sont plus que jamais confrontés au manque d'hommes du fait de la défection croissante des troupes supplétives, constituées par les prisonniers de guerre soviétiques et les habitants locaux qui, sentant la victoire imminente des Soviétiques, profitent de la confusion croissante pour passer dans le camp des partisans. Les EKA et les cavaliers recrutés parmi les prisonniers de guerre (Ostreiter) sont donc progressivement désarmés par les Allemands. ${ }^{36}$

Quant aux hommes qui continuent à combattre sous l'uniforme allemand, leur comportement est de plus en plus incontrôlable. Le commandement édicte ainsi ses instructions au sujet des mesures adéquates à prendre vis-à-vis des civils, en fonction de leur degré d'hostilité.

Le comportement des troupes allemandes à l'encontre de la population qui n'a pas sympathisé avec les bandes s'est avéré à certains endroits sans ménagement. La population a été dépossédée de tous ses biens du fait des réquisitions et du pillage, si bien qu'elle reconnaît ouvertement préférer passer du côté des bandes, car ces dernières leur laissent assez pour vivre. Ces cas doivent donc être condamnés de façon particulièrement ferme, car des soldats allemands se sont ici personnellement enrichis avec l'accord tacite de leur chef d'unité. ${ }^{37}$

L'épisode suivant, relaté moins d'un an après les faits, en septembre 1944, par une habitante de Monastyrsk, témoigne de cet état de déréliction :

Le 15 novembre 1943 dans notre village de Monastyrsk sont arrivés quelques Estoniens de la garde allemande de la voie ferrée de la gare de Verebenka, tous soûls, qui ont ouvert le feu à l'aveuglette dans toutes les directions, sur les maisons, tuant la citoyenne Ivanova Domna Larenovna. Une fois qu'ils eurent cessé et quitté le village de Monastyrsk, j'ai vu la citoyenne Ivanovna D.L., abattue à côté de la cabane de son sauna, avec plusieurs balles dans la poitrine. [...] Le même jour où ils ont tué la citoyenne Ivanova D.L. les Estoniens ont pillé les objets de valeur chez les habitants du village de Monastyrsk et les ont emportés avec eux. ${ }^{38}$

35. GARF, f. 7021, op. 30, d. 250a, 1. 201-202.

36. BA-MA RH26-207/23.

37. BA-MA, RH26-207/24a, Anlage 177.

38. GARF, f. 7021, op. 30, d. 250a, 1. 242-242 ob. 
La chasse à l'homme dans les villages et à travers les bois s'accompagne des pires cruautés: les habitants trop vieux ou trop malades pour s'enfuir des villages désertés, sont abattus, torturés, brûlés vifs.

Les déportations de masse frappent donc une seconde fois les familles. Cependant, à la différence du printemps 1942, l'ensemble des habitants, y compris les enfants, et les personnes invalides sont embarqués. Ainsi de la famille Gagarin du village de Vjazovoe, Pol’skij sel'sovet.

Le 6 janvier 1944, un détachement punitif allemand a encerclé dans la forêt le camp où se cachait la population. Ils ont emmené de force ma mère Anastasija Vasil'evna Gagarina, née en 1898, mon frère Evgenij Georgievič Gagarin, né en 1936, ma fille Larisa Anatol'evna Mihailova, née en 1943 et ils les ont déportés en Allemagne. Je ne sais rien de ce qu'il leur est arrivé par la suite. 25 août 1944, signé Gagarina. ${ }^{39}$

En conclusion de son témoignage recueilli en août 1944, Vera Georgievna Gagarina ajoute : «Je ne sais rien de ce qui leur est arrivé par la suite ». Ces événements dramatiques, à quelques jours de la libération, ont été précédés d'autres épisodes tragiques pour cette famille. Alors que les villageois de Vjazovoe se cachent dans les bois depuis octobre, une sœur de Vera, Marja, sortie du camp un jour de novembre pour tenter de récupérer des vivres dans le village, tombe dans une embuscade d'Estoniens et est exécutée. L'année précédente, en mai 1942, un autre frère de Vera, Viktor Georgievič Gagarin avait fait partie des membres du kolkhoze « Probuditel' » déportés en Allemagne. Les Gagarin sont ainsi une fratrie parmi tant d'autres décimées par les déportations successives, comme l'atteste l'examen des listes de déportés dressées à la libération.

La violence de l'occupant combinée avec l'avancée de l'Armée rouge conduit au basculement, de gré ou de force, de la population civile du côté des partisans.

Selon les sources allemandes, les partisans enrôlent de force les villageois dans leurs rangs. Nous savons que l'attitude des partisans vis-à-vis des civils n'est pas dénuée de violence ${ }^{40}$. Les relations entre civils et partisans n'ont pas pour seul objet l'enrôlement des premiers, elles se nouent également autour de l'aide apportée aux seconds, sous la forme d'informations sur les forces ennemies ou encore du soutien matériel, ce qui, d'ailleurs, constitue un aspect essentiel du fonctionnement du mouvement partisan depuis ses débuts ${ }^{41}$. La consignation de cette aide refera surface à la libération, à l'aide des reçus laissés par les partisans. Cependant, avec quel degré de consentement les villageois se dépouillèrent-ils de leur bétail, de grain et de vêtements pour aider les partisans ? L'ambivalence des sources, interdisant une réponse formelle, montre bien l'enjeu de cette question : tandis que les

39. Témoignage de Gagarina Vera Georgievna, village de Bjazov, Pol'skij sel'sovet, GARF, f. R-7021, op. 30, d. 250a, 1. 153.

40. Cf. article de Masha Cerovic dans ce même numéro.

41. Une partie des formations initiales de partisans périssent ou disparaissent, victimes de la famine au cours de l'hiver-printemps 1942. 
sources allemandes évoquent essentiellement des raids de pillage de la part des partisans terrorisant les villageois, les témoignages pour la Commission soviétique évoquent quelques mois plus tard le martyre de ceux, souvent des femmes, qui furent dénoncés aux Allemands pour avoir donné de la nourriture aux partisans ${ }^{42}$.

Les habitations elles-mêmes peuvent être détruites par les partisans. Alors que la pression de ces derniers s'accentue fortement depuis l'été 1943, les combats les opposent aux troupes allemandes et à leurs supplétifs dans les villages désertés. La mention, dans les sources allemandes, de villages incendiés par les partisans ne dit rien des circonstances et des raisons de ces actes ${ }^{43}$. «Dans le district de Slancy, tous les villages sauf 4 ont été détruits, dont une partie par les bandits $»^{44}$, résume un officier allemand au début de décembre 1943.

Si elles différent sur les auteurs des destructions, les sources russes et allemandes s'accordent donc sur le degré de dévastation de la région dans ces combats sans merci des derniers mois de la guerre qui transforment le district de Slancy en une région totalement sinistrée.

La présence croissante des partisans ne se traduit pas seulement par la recrudescence des combats, mais aussi par la reprise en main de la population civile. À partir de l'automne fonctionnent des orgtrojki, dont les rapports d'activité envoyés à l'état-major partisan à Leningrad respirent la langue bureaucratique soviétique. Audelà du travail de propagande pour tenter de gagner l'adhésion et s'assurer de la fiabilité des habitants, la troïka organise le transfert des populations sinistrées dans des camps de zemljankit établis dans les bois et tentent de fournir un peu de vivres aux plus démunis d'entre eux. En contrepartie, les partisans prélèvent les denrées nécessaires à l'entretien de leurs propres troupes sur une population déjà à la limite de la famine. Dans cette perspective de gestion de la population, la troïka entreprend également le premier travail d'inventaire des gigantesques pertes subies par le district, notamment le nombre des maisons incendiées ${ }^{46}$.

\section{Restauration}

\section{Une paysannerie exsangue}

Le caractère désertique à l'échelle de l'ensemble de la région de Leningrad frappe les premiers représentants non partisans du pouvoir soviétique, qui consignent dans

42. Les cas de pillage par de petites unités de partisans sont également évoqués, pour les condamner, dans les archives des partisans. Cf. Hill, The War in the Eastern Front..., p. 145.

43. Il s'agit des villages suivants: Samosčie, Salesčie, Lučki et Popkova Gora, BA-MA, RH 26-207/23, p. 268.

44. BA-MA, RH26-207/23, p. 384, Notizen über die Besprechung des Div.-kdr mit SR 94 in Gdow am 8.12.43.

45. Zemljanka désigne une habitation de fortune, sorte de hutte en bois à moitié ensevelie dans la terre.

46. Matériaux conservés au musée de Slancy. 
leurs rapports sur la libération les villages désertés et incendiés, les villes rasées et vidées de leurs habitants Progressivement, la population sort des bois, rendant le travail d'enregistrement très fragile. Dans Kingissepp en ruines (90 \% des constructions de la ville ont été détruites), les Soviétiques ont enregistré, au 1er février 1944, 650 personnes en tout et pour tout. Quelques jours plus tard, le 7 février, 1591 personnes sont déjà décomptées, dont 352 hommes, 929 femmes, mais dans le district alentour, pas âme qui vive dans six sel'sovety dont toute la population a été évacuée par les Allemands. Au même moment à Gačina, qui comptait 38200 habitants à la veille de la guerre, 3595 personnes sont enregistrées, dont 748 hommes et 2105 femmes. À Volosovo, les Allemands n'ont pas achevé leur travail de destruction des bâtiments. Ils ont également laissé derrière eux 600 évacués en provenance de Gačina, Puškin, Krasnoe Selo, Uritsk. À chaque fois, la poignée d'hommes en âge et aptes à servir est envoyée à l'armée, tandis que recommence une mobilisation intensive pour déblayer les ruines, bâtir à la hâte, remettre en route l'activité locale ${ }^{47}$.

Quant au district de Slancy, sur les 15700 habitants d'avant-guerre, on en recense 5848 au 25 février 1944, dont 1921 ont moins de 14 ans. 1319 ne sont pas des locaux, mais pour la plupart des personnes évacuées par les Allemands, en provenance de différents districts de la région, encore plus démunis que les locaux, la moitié d'entre eux ne disposant d'aucune réserve alimentaire. Au 20 mars, on a trouvé 5324 personnes, dont 1152 hommes, 3003 femmes, 1169 enfants de moins de 14 ans. 270 personnes ont été déclarées aptes à servir, 2353 en état de travailler ${ }^{48}$.

\section{La population du district de Slancy au 21 février 194449 :}

\begin{tabular}{|l|c|c|c|c|}
\cline { 2 - 5 } \multicolumn{1}{c|}{} & Hommes & Femmes & Enfants & Total \\
\hline Population locale & 904 & 2026 & 1217 & 4147 \\
\hline Réfugiés & 180 & 639 & 500 & 1319 \\
\hline Total & 1084 & 2665 & 1717 & 5466 \\
\hline
\end{tabular}

Les fluctuations des chiffres reflètent les mouvements de population liés au départ des évacués et, à l'inverse, au retour de certains habitants réfugiés à proximité.

47. CGA SPb (Central'nyi Gosudarstvennyj Arkhiv Sankt Peterburga - Archives d'État centrales de Saint-Pétersbourg), f. 7179, op. 53, d. 89, 1. 4-5ob, cité in A.R Dzeniskevič, red., Iz rajonov oblasti soobščajut... Svobodnye ot okkupacii rajony Leningradskoj oblasti $v$ gody Velikoj Otečestvennoj vojny, 1941-1945: sbornik dokumentov [Communiqués des rajony de l'oblast... Les rajony de l'oblast de Leningrad libérés de l'occupation pendant la Grande Guerre patriotique, 1941-1945 : recueil de documents], SPb : DB, 2006, p. 416-420. CGAIPD $\mathrm{SPb}$ (Central'nyj Gosudarstvennyj Arhiv Istoriko-Političeskih Dokumentov Sankt Peterburga - Archives d'État centrales de documents historiques et politiques de Saint-Pétersbourg), f. 24, op. 5, d. 6072 .

48. LOGAV, f. 624, op. 1, d. 10, 1. 8-9, 1 ; CGA SPb, f. 1684, op. 5, d. 837, 1. 92-99, cité in Iz rajonov oblasti ..., p. 442.

49. Musée de Slancy, «Vsego naselenija po Slancevskomu rajonu na 21 fevralia 1944g. » [La population du district de Slancy au 21 février 1944]. 
Cependant, ces données incertaines décrivent toutes la même réalité : une population diminuée de près du tiers par rapport à l'avant-guerre, constituée pour moitié d'une population adulte en état de servir et surtout de travailler, pour $22 \%$ d'enfants et près d'un tiers $(29 \%)$ de personnes inaptes au travail car trop âgées ou malades. Une fois partis sous les drapeaux les quelques hommes jeunes encore présents au moment de la libération, il reste essentiellement pour reconstruire le district des femmes avec leurs enfants et des personnes âgées ou invalides. Il n’y a encore aucune nouvelle des centaines de personnes, la plupart jeunes, déportées pour le travail pendant l'occupation, soit 844 personnes, pour moitié il est vrai des réfugiés en provenance des districts proches de Leningrad ${ }^{50}$.

Dans ce district sinistré, la tâche est donc immense et les ressources quasi inexistantes.

L'ampleur des destructions est sans appel, même si, là encore, les chiffres peuvent fluctuer quelque peu : sur 103 localités, 78 ont été totalement détruites, 6 partiellement. 1637 maisons de kolkhoziens ont été anéanties, il n'en reste que 466 debout. Les villageois continuent donc, et pour longtemps, de s'abriter dans des zemljanki dans les bois, tandis que 2000 personnes environ s'entassent dans les quelques maisons à peu près intactes : 854 personnes dans le sel'sovet Novosel'skij, 1000 dans le Rudnenskij, 223 dans le Pol'skij. Les sel’́sovety Černovskij, Vyskatskij, Savinovšenskij et Pelešskij sont rasés. Dans le bourg de Slancy luimême, détruit à plus de $80 \%$, où se trouvent encore près de 600 personnes, 216 maisons et immeubles d'habitation (soit 1372 pièces d'habitation) sont en ruines ${ }^{51}$.

Privés de toit, les villageois manquent de tout, à commencer par le bétail. Un rapport indique que les Allemands ont emmené près de 1000 têtes de bétail. De toute évidence, ce chiffre ne recouvre que la période s'écoulant entre la fin 1943 et le début de 1944, lors de l'évacuation de la région par les Allemands. De fait, ceux cités dans une réunion du parti du district sont bien plus élevés : plus de 2000 têtes de gros bétail tuées ou emportées, plus de 500 chevaux, et 2422 têtes de petit bétail $^{52}$. Au moment de sa libération, il ne reste plus dans tout le district que 370 chevaux, 652 vaches, 292 moutons, 356 chèvres, 3 cochons. Au début du mois de mars, le président du comité exécutif cherche à obtenir auprès des autorités régionales de Leningrad des nouvelles du bétail évacué au début de la guerre, et dont il a perdu la trace, passé la ville voisine de Kingissepp ${ }^{53}$.

Tout comme la rareté de la main-d'œuvre, la quasi-absence d'animaux source d'alimentation de base et force de traction indispensable aux cultures - , tourne à l'obsession chez les nouveaux dirigeants, le bétail occupant une place

50. Musée de Slancy, Spravka o položenii v Slancevskom rajone v period okkupacii nemeckofašistskimi vojskami [Rapport sur la situation dans le district de Slancy pendant l'occupation par les troupes germano-fascistes].

51. LOGAV,f. 624, op. 1, d. 10,1.8-9.

52. CGAIPD SPb f. 24, op. 5, d. 6272,1. 13.

53. LOGAV, f. 624, op. 1, d. 10,1. 13. 
centrale comme objet de litige mais aussi de transaction entre les pouvoirs publics et les particuliers.

La station de machines agricoles (Rudnenskaja MTS) est remise sur pied à partir de mars par son ancien directeur mais les tracteurs ont été emmenés par les Allemands et ceux envoyés par Leningrad se révèlent de piètre qualité.

\section{Notabilités locales}

Ce sont les partisans qui libérèrent Slancy, et ce sont les hommes issus des rangs des partisans qui prirent les fonctions de responsabilité dans le district. Le secrétaire du comité du parti au niveau du district, I.G. Svetlov, est commandant de la 9e brigade, le secrétaire chargé des cadres du parti, Mihajl Alekseevič Gubernatorov, a combattu auprès de S.N. Pirogov 54 .

Le président du comité exécutif du district, Mihail Osipovič Ivanov, travaillait comme agronome dans une MTS du district avant-guerre. Trois fois décoré pendant la guerre de partisans, il préside pendant plusieurs années à la lourde tâche de remettre sur pied un district en ruines ${ }^{55}$. La plupart des personnes qui entourent Ivanov font partie de la poignée de communistes du district. Tout comme Ivanov, certains sont d'anciennes figures locales. C'est, par exemple, le cas du directeur de la MTS, A.G. Lučin dont la femme a été fusillée par les Allemands en 1942, et dont la fille, Lilija Aleksandrovna, est secrétaire du Komsomol et assesseur au tribunal populaire ${ }^{56}$.

Leur poids dans la vie du district n'est cependant pas toujours clair. D'une part, au sein de la hiérarchie de la région de Leningrad, la priorité ne va pas forcément aux habitants du district de Slancy, ce dont se plaint Ivanov, face à ses demandes laissées sans réponse en matière d'approvisionnement. Ensuite, au sein même du district, il fait remarquer combien la faiblesse de la rémunération des personnes au service du comité exécutif rend la fonction peu attractive. La maigreur des ressources tout comme le dénuement dans lequel se trouvent les dirigeants du district sont des facteurs importants à prendre en considération sachant qu'une de leurs tâches essentielles est la distribution des biens de première nécessité et de l'aide matérielle aux habitants. Ainsi, en décembre 1944, Ivanov se plaint-il du manque de cadres aussi essentiels pour le district que des agronomes et des vétérinaires compétents, alors qu'il a peu à leur offrir, pas même de quoi les vêtir et les chausser correctement, tandis que les salaires proposés sont très bas, comparés à ceux en vigueur dans l'appareil du parti, mais aussi dans les combinats industriels réinstallés dans le bourg. Une dactylo du comité exécutif reçoit 160 roubles quand

54. Sur la désignation des premiers responsables du district, dans une maison de Bol’šie Polja, le 6 février 1944, cf. V.V. Ivanov, Slancy : Istoriko-kraevedčeskij očerk [Slancy : essai historique régional], L. : Lenizdat, 1988.

55. CGAIPD SPb, f. 24, op. 5, d. 6272,1.17-21.

56. LOGAV, f. 624 , op. 1, d. 13,1. 66 . 
elle peut en espérer 450 en travaillant pour l'entreprise Leningradslanec ou Leningradstroj ${ }^{57}$.

Le second cercle est constitué par les dirigeants des soviets ruraux et les présidents de kolkhoze. Malgré la diminution spectaculaire de la population, les huit sel'sovety d'avant-guerre sont reconstitués, le territoire du district faisant l'objet par la suite de modifications territoriales : en janvier 1945, deux sel'sovety se trouvant en territoire estonien, Zagriv'e et Ust'-Černo, sont rattachés au district de Slancy, alors que leur population est pour l'essentiel toujours réfugiée dans les districts voisins de Kingissepp et Volosovo ${ }^{58}$. Au nombre de 65 avant-guerre, 59 kolkhozes sont rétablis sur le papier, ce qui signifie des effectifs extrêmement réduits au sein de chacun d'entre eux.

Il faut nommer en priorité des personnes ayant participé au mouvement partisan et ayant apporté de l'aide aux partisans aux postes de présidents des sel'sovety et des kolkhozes..$^{59}$

Cette déclaration ne constitue pas tout à fait un vœu pieux. Pendant tout le mois de février 1944, les dirigeants du district établissent des listes pour classer les habitants en fonction de leur attitude pendant la guerre, telle cette liste de 38 personnes ayant activement travaillé dans les organisations clandestines du district de Slancy. Bien plus étoffée, une liste datée du 10 février 1944 fixe le nom de 135 personnes ayant apporté de l'aide aux partisans pendant toute la durée de l'occupation ${ }^{60}$. D'autres listes tentent à l'inverse d'établir le nom des traîtres (predateli), des anciens policiers, des personnes parties avec les Allemands, etc. Établies au niveau des sel'sovety ou des kolkhozes, elles témoignent du travail d'épuration et de dénonciation qui s'effectue au moment de la libération.

Certains noms figurant sur les «bonnes » listes d'habitants dressées en février se retrouvent donc parmi les présidents de kolkhozes, membres des sel'sovety ou encore assesseurs ou présidents des tribunaux populaires narsudy. Par exemple, Pavel Terent'ev, agent des impôts avant la guerre, fait partie de la poignée d'habitants du district membres d'organisations clandestines pendant la guerre, et se révèle être un pilier local après-guerre comme président du sel'sovet Pelešskij, de même que Klavdija Beljaevskaja, institutrice avant-guerre, adjointe du juge du

57. CGA SPb, f. 7179, op. 14, d. 26, 1. 176-177 ob. Cité in Iz rajonov oblasti..., p. 524. L'absence de moyens matériels contribue également à miner les efforts de l'exécutif : « hotja i meloč', no dlja nas trudnost' - eto kanceljarskie pribory, kak-to: černil'nye pribory, presspap'e, nastol'noe i pročee oborudovanie." [Les fournitures de bureau, ce n'est pas grand-chose et pourtant c'est difficile, qu'il s'agisse de stylo, de presse-papier ou de n'importe quelle autre fourniture], Ibid.

58. LOGAV,f. 624 , op. 1 , d. $45,1.1$.

59. CGA SPb, f. 1684, op. 5, d. 837, 1. 92-99, cité in Iz rajonov oblasti..., p. 445.

60. Musée de Slancy, «spisok aktivno rabotajuščih v podpol’nyh organizacii Slancevskogo rajona » [Liste des travailleurs actifs dans les organisations clandestines du rajon de Slancy] (6 d'entre eux sont des habitants du district voisin de Osmin, les autres sont de Slancy ou des sel'sovety du district) et «spisok lic okazyvajuščih pomošči partizanam s $16.07 .41 \mathrm{~g}$. po $1.02 .44 \mathrm{~g} »$ [Liste des personnes ayant aidé les partisans entre le 16.07.41 et le 1.02.44]. 
district en 1944 ou Antonina Antonova, également institutrice, assesseur au narsud mais qui, plus jeune d'une vingtaine d'années, participe également à la constitution d'une nouvelle cellule de komsomols ${ }^{61}$. Les personnes les plus instruites et les plus loyales au régime figurent dans l'organigramme, à l'instar d'Anna Nikolaevna Lisakova, institutrice âgée alors d'une vingtaine d'années, élue membre du sel'sovet Novosel'skij au printemps 1944, et assesseur du tribunal populaire, ou encore du grand homme du district, Mihail Ivanovič Pimenov, combattant de la guerre civile, partisan blessé à plusieurs reprises, qui redeviendra président du kolkhoze «Voshod » tout en étant élu député au Soviet Suprême en 1946. Cependant, il s'agit avant tout de trouver des personnes capables d'organiser le quotidien des ruraux, parmi la poignée d'adultes dispersés dans chaque village.

La reconstitution d'une hiérarchie locale va de pair avec le travail d'inventaire organisé par les Soviétiques pour faire le bilan de l'occupation. Ce travail s'opère dans différentes directions, sous l'égide de la Commission d'enquête soviétique. Présidents de sel'sovety et de kolkhozes organisent dans ce cadre la collecte des inventaires et des témoignages. Commencé dès le printemps 1944, ce travail s'étire ensuite sur de longs mois, au rythme des retours de la population réévacuée et rapatriée. Les commissions locales sont composées de ces « notables » locaux, qui ont été eux-mêmes victimes de l'occupation. Il en va ainsi de la commission pour le sel'sovet Pol'skij, qui compte trois membres, dont Dar'ja Georgievna Petuhova, présidente du sel'sovet et Lidija Vasilievna Šumilova, secrétaire du sel'sovet. Dans l'acte établissant les crimes commis par l'occupant dans le sel'sovet qu'elles signent toutes deux le 10 novembre 1944 figurent plusieurs membres de leurs familles, y compris des frères et des sœurs, parmi les jeunes kolkhoziens déportés du travail. Dar'ia compte également un frère, fusillé en 1941 pour avoir voulu sauver l'honneur d'une autre sœur, aux prises avec des officiers allemands au cours d'une beuverie nocturne ${ }^{62}$.

Tandis que se reconstitue la hiérarchie du district, les combats se poursuivent sur son territoire pendant tout le printemps et l'été 1944. L'Armée rouge y a pris ses quartiers ${ }^{63}$, avec ses hommes, ses constructions de fortune pour les loger, ses chevaux. L'armée est une source d'aide pour les paysans dans le récit officiel. C'est sous ses ordres, et à l'aide de sa logistique, que les villageois s'attellent à la restauration des routes, des ponts, de la voie de chemin de fer, puis des préparatifs aux semailles de printemps. Cependant, loin de reprendre le cours de leur vie, une bonne partie des rescapés du district doivent à nouveau quitter leurs villages, compris dans la zone de front. Ils sont donc évacués dans les kolkhozes des sel'sovety plus au sud, davantage épargnés. En mai, sont ainsi évacués (otselenie)

61. Musée de Slancy, «spisok aktivno rabotajuščih v podpol’nyh organizacii Slancevskogo rajona » et «spisok lic okazyvajuščih pomošči partizanam s 16.07.41g. po 1.02.44g. »; LOGAV, f. 624, op. 1, d. 13, 1. 111, d. 70, 1. 35 et 203 (Terent'ev) ; 1.65-66 (Beljavskaja); 1. 66, 134 (Antonova).

62. GARF, f. 7021, op. 30, d. 250,1. 147-148; 173, 173ob, 174.

63. Il s'agit de 1'état-major de la 2e Armée de choc sous les ordres du lieutenant-général I.I. Fedjunin, cantonné à Bol’šie Polja, cf. Ivanov, Slancy... 
les villageois des sel’́sovety Pelešskij et Pol’skij, dirigés vers les kolkhozes des sel'sovety Popkogorskij, Rudnenskij, Vyskatskij. En juin, plusieurs kolkhozes des sel'sovety Pokogorskij et Vyskatskij, dont ceux qui avaient accueillis les évacués de mai, ainsi que des kolkhozes Černovskij sont évacués vers les localités des sovets Rudnenskij, Savinovščinskij, Novosel’skij. Les institutions du district sont elles-mêmes dispersées entre plusieurs villages. Malgré les dispositions prises à l'égard des biens collectifs et personnels, cette évacuation constitue pour les habitants une sérieuse menace de perdre ce qui a été miraculeusement préservé pendant l'occupation. De fait, même si elle ne se déroule pas dans le climat de terreur de l'automne 1943, elle suscite l'inquiétude des autorités locales et des habitants confrontés à nouveau au risque de retrouver leurs biens détruits, ou volés par la troupe ${ }^{64}$. Ce n'est qu'en octobre que les habitants reviennent sur leurs terres et que Slancy sert définitivement de cadre aux institutions du rajcentr, c'est-à-dire des départements du soviet exécutif local.

En décembre 1944, des funérailles officielles sont organisées pour enterrer une seconde fois les partisans et soldats du district morts sous les balles de l'ennemi et réunis dans un même tombeau. À la fin de l'année 1945, deux monuments, l'un en mémoire des combattants de l'Armée rouge, l'autre en l'honneur des partisans, sont dessinés par l'architecte académicien Nikol'skij65.

\section{Restaurer le système du kolkhoze}

Derrière le culte rendu à l'héroïsme local se cache la profonde inquiétude des dirigeants locaux face au degré de loyauté des paysans confrontés à la restauration du régime soviétique, et au premier chef au système des kolkhozes. « Il reste des traces parmi la population de l'administration économique allemande, elles vont se voir dans la conduite des semailles, tous les communistes doivent en tenir compte $»{ }^{66}$ est-il déclaré lors d'une réunion des organisations du parti pour le district, le 29 avril 1944. On s’y plaint également de la faiblesse de la propagande auprès de la population. Les questions posées au cours de rassemblements populaires organisés dans la région et notamment dans le district de Slancy, dans lequel pas moins de 52 exposés (doklady) et réunions de discussions (besedy) auraient été tenus au cours du premier mois de sa libération, sont très édifiantes sur l'état d'esprit des locaux.

(Outre des questions sur la situation sur le front), la population assistant aux réunions de discussions, aux exposés et conférences [tenus par les propagandistes du comité du parti de l'oblast de Leningrad, notamment dans les districts

64. Cf. la plainte du président du comité exécutif du district voisin de Sošihinskij au Voennyj Sovet Lenfronta sur les agissements illégaux d'une partie de la troupe, 30 avril 1944, CGA $\mathrm{SpB}$, f. 7179 , op. 53, d. 90, 1. 19, cité in Iz rajonov oblasti..., p. 456.

65. LOGAV, f. 624, op. 1, d. 13,1. 134 ; f. 624, op. 1, d. 47,1. 182.

66. CGA IPD SPb, f. 24, op. 5, d. 6272,1.1-3. 
de Volosovo, Gačino, Krasnosel'skij, Slancy], a posé des questions du type suivant $[\ldots]$ : « la charte des kolkhozes a-t-elle été modifiée ?», «Les enfants nés de pères allemands recevront-ils les droits des citoyens soviétiques?», «Est-il vrai que les relations entre le gouvernement soviétique et l’Église ont changé ? », etc. ${ }^{67}$

Ces questions attestent, sinon de l'hostilité, du moins de la fragilité des positions communistes : notamment en ce qui concerne les kolkhozes, le retour au système d'avant-guerre n'a rien d'évident pour les habitants des régions libérées, alors que pour les autorités il est pleinement à l'ordre du jour ${ }^{68}$.

La crainte que les agents de l'occupant ne soient restés sur place, instillant un état d'esprit hostile au retour des autorités et des institutions soviétiques au sein de la population, est à nouveau formulée par le camarade Smirnov, du NKGB local, lors d'une réunion du parti du district de Slancy, le 13 août 1944 :

Nous vivons dans un district où les fascistes ont mené pendant deux ans et demi leur agitation fasciste, nous ont laissé leurs agents qui se trouvent jusqu'à maintenant dans plusieurs kolkhozes et organisations. Nous tous les communistes devons les démasquer et prendre des mesures pour leur isolation. ${ }^{69}$

La tâche des autorités communistes est donc double : opérer un travail de rééducation idéologique face à une paysannerie qui a pu considérer positivement certaines modifications introduites par les Allemands et effacer les traces de l'occupation dans le statut de propriété d'un certain nombre de biens. Le kolkhoze doit ainsi récupérer terres et bétail qui se sont retrouvés, par différentes voies, chez les particuliers qui se les sont appropriés. Une des premières mesures consista dans la redéfinition des limites des terres collectives et des lopins. À la fin de l'été 1944, toutes les terres du district devaient être remesurées, les documents cadastraux remis à jour et soumis à l'approbation de l'assemblée des kolkhoziens, afin de rendre aux kolkhozes les terrains rattachés pendant l'occupation aux lopins privés, les bornes marquant la frontière entre les deux types de propriété devant être replantées ${ }^{70}$.

En ce qui concerne le bétail, le travail prit plusieurs mois pour obtenir des citoyens indélicats qu'ils restituent les vaches entrées en leur possession sous l'occupation. Les conflits autour des vaches, qui affectent l'ensemble des territoires libérés soviétiques,

67. TsGA SPb, f. 1684, op. 5, d. 837, 1.92-99, cité in Iz rajonov oblasti..., p. 447. Le rapport ne portant pas uniquement sur le district de Slancy, il est possible que certaines questions reflètent davantage les préoccupations d'autres districts, notamment en ce qui concerne les enfants nés de soldats allemands. Ainsi, la fréquentation de femmes russes par l'occupant est avérée pour la ville de Gačina, en partie du fait de la présence de maisons closes pour la troupe. Le fait est plus douteux pour le district de Slancy.

68. Ce point n'est qu'une nouvelle illustration du thème des « espoirs déçus », brillamment développé par Elena Zubkova.

69. CGA IPD SPb, f. 24, op. 5, d. 6272,1. 11.

70. LOGAV, f. 624, op. 1, d. 13,1.48-49. Sur la nouvelle campagne de vérification de la taille des lopins en 1946, cf. Jean Lévesque, « Part-time Peasants : Labour Discipline, Collective Farm Life and the Fate of Socialist Agriculture... ». 
posent deux questions essentielles. La première est celle du statut de propriété : à qui appartenait une vache faisant partie du cheptel du kolkhoze avant-guerre et qui s'était retrouvée entre les mains d'un particulier pendant l'occupation? Le type de transaction (forme de rétribution par les Allemands, achat, partage sauvage du cheptel) faisait-il une différence ${ }^{71}$ ? La seconde question porte sur la mise en œuvre d'une justice sociale informelle dans le contexte local soviétique. Plusieurs pôles d'autorité au niveau local, dont l'assemblée des kolkhoziens sont alors en jeu. Loin d'être une simple chambre d'enregistrement, elle prend des décisions visant sans doute à protéger certains de ses membres les plus démunis. Cependant, en cas de conflit, le dernier mot revient au comité exécutif ${ }^{72}$. Ainsi, dans plusieurs kolkhozes, l'assemblée des kolkhoziens concède à certains de ses membres, sans doute particulièrement dans le besoin, la jouissance d'une vache appartenant de droit au kolkhoze. Le comité exécutif tranche à son tour, généralement dans le sens d'un respect strict de la propriété du kolkhoze : la réussite du plan passe avant la commisération, quitte à recommander à la personne dépossédée de se tourner vers le narsud. C'est le conseil que donne le comité exécutif à la citoyenne Nikiforova A., habitante du village Ryžikovo, sel'sovet Rudnenskij, qui doit rendre une vache appartenant au kolkhoze « Burevestnik » sel'sovet Vyskatskij. Apparemment, elle a été dupée par la citoyenne Knudi O., du village de Gverezdno, Novosel'skij s/sov, qui lui a vendu une vache appartenant en réalité au kolkhoze ${ }^{73}$.

En janvier 1945, le comité exécutif statue sur le sort de 18 vaches qui se trouvent entre les mains de particuliers depuis l'occupation. La plupart d'entre elles sont destinées à retourner dans les étables du kolkhoze. Quelques arrangements sont cependant proposés : le comité exécutif suggère à l'assemblée des kolkhoziens d'accepter de reprendre un veau (telka, telenka, netel`), en laissant la vache au kolkhozien, souvent kolkhozienne, lorsque celle-ci est décrite dans une «situation matérielle difficile », ou encore de lui fournir du lait ou de lui proposer un veau à l'achat. Quant aux vaches réputées « sans maître », c'est-à-dire ayant appartenu à des personnes privées mais retombées dans l'escarcelle publique - généralement suite à une confiscation ou au départ/disparition de son propriétaire - , elles peuvent servir aux bonnes œuvres du comité exécutif : celle de Burmistrova du village Bol'šie Polja est vendue au prix d'État (po gosudarstvennoj stoimosti) par le département des Finances au camarade Pavlov D.S., agronome principal du département Agriculture, néanmoins malade et plongé dans une situation matérielle décrite comme critique ${ }^{74}$. Ce prix d'État, s'il

71. Les affaires relatives à la propriété des vaches, korovye dela, font l'objet de plusieurs articles dans la presse juridique spécialisée de l'époque.

72. Pour la mise en scène des relations entre les assemblées de kolkhozes et le comité exécutif de district, cf. Fedor Belov, The History of a Soviet Collective Farm, New York : Praeger, 1955.

73. LOGAV, f. 624, op. 1, d. 13, 1. 69ob.

74. LOGAV, f. 624 , op. 1, d. 60. Burmistrova fait partie des personnes à qui on supprime des avantages pour données compromettantes (f. 624, op. 1, d. 13,1. 60ob.). Une Burmistrova, sans doute parente, figure au printemps 1945 parmi les kolkhoziens primés du sel'sovet Pol'skij, f. 624 , op. 1 , d. $45,1.144$. 
représente une somme importante, reste néanmoins en dessous du prix du marché. La citoyenne Petuhova, déjà évoquée, obtient de pouvoir racheter pour 1500 roubles la vache de son prédécesseur au poste de président du sel'sovet Pol'skij, limogé pour indélicatesse vis-à-vis de biens qui lui avaient été confiés ${ }^{75}$. En revanche, la décision du 28 février 1945 de donner la vache confisquée de la citoyenne E.I. Pavlova au camarade Egorov, instructeur au comité de district du parti, est invalidée quelques mois plus tard, pour violation du décret du SNK SSSR du 17 avril $1943^{76}$.

Les désordres liés à l'évacuation de 1941 et à l'occupation se reflètent également dans la demande du comité exécutif à l'égard des citoyens qui se sont emparés de meubles, lors du pillage de diverses administrations et de logements des évacués des bourgs de Slancy et de la mine $n^{\circ} 2$. Les villageois indélicats sont invités à rendre avant le 20 octobre 1944, sous peine de saisie et de poursuites judiciaires, ces biens mal acquis : des lits, matelas, chaises, miroirs, armoires, objets de base de la civilisation urbaine, mais qui sont des raretés dans les villages ${ }^{77}$.

Un autre changement de statut des biens, caractéristique de la sortie de guerre en milieu rural, concerne le sort des biens des personnes considérées comme des collaborateurs. Que leurs propriétaires se soient enfuis avec les Allemands ou qu'ils aient été jugés et condamnés ${ }^{78}$, ces biens sont confisqués et, passant dans l'escarcelle publique, sont répartis entre les institutions régies par le comité exécutif ou partiellement distribués aux nécessiteux du district ${ }^{79}$.

Dans quelle mesure cette remise sur pied du système des kolkhozes, à vrai dire peu modifié par l'occupant, s'accompagna-t-elle de résistances, frictions, conflits, comme le craignaient les autorités locales au printemps 1944 ? Dans quelle mesure le comportement de chacun pendant la guerre influa-t-il sur son sort, ou celui de sa famille après la guerre ? Nous verrons plus loin qu'il est difficile de répondre de façon tranchée à ces questions, mais il apparaît clairement que les faits et gestes de chacun sont connus, ce qui n'a rien d'étonnant dans une communauté au nombre relativement réduit. Le poids des interconnaissances semble ici fondamental et ces dernières sont sans cesse mobilisées dans la constitution de listes d'habitants, comme nous l'avons vu plus haut. Qu'on considère par exemple la liste des citoyens s'étant emparé de meubles pendant l'occupation: elle comporte une cinquantaine de noms de villageois et la consignation du nombre exact et de la nature des biens à rendre ${ }^{80}$.

75. LOGAV, f. 624, op. 1, d. 13,1. 61.

76. LOGAV, f. 624 , op.1, d. $45,1.32,67$.

77. LOGAV, f. 624, op. 1, d. 13,1.69, 74-74 ob.

78. Cf. dans le même numéro, Vanessa Voisin et Sergey Kudryashov.

79. LOGAV, f. 624, op. 1, d.13, 1. 21. Le parallèle est frappant avec le sort des biens des ruraux dékoulakisés dans les années 1930.

80. LOGAV, 624, op. 1, d. 13,1. 74-74 ob. 


\section{Distribution}

\section{Listes}

Les discriminations à l'égard des personnes considérées comme compromises avec l'occupant sont difficiles à établir avec précision, en raison de l'impossibilité d'accéder aux archives relatives aux activités de l'ONKVD, l'ONKGB et du Rajkom VKP(b) du district. Elles apparaissent simplement en filigrane dans les décisions du comité exécutif. Toutefois, d'autres critères interviennent dans le travail incessant de catégorisation auquel se livrent les autorités du comité exécutif et, au premier chef, celles en charge de l'aide sociale. Celles-ci dressent des listes de bénéficiaires d'une aide qui prend plusieurs formes : distribution de numéraire, mais aussi en nature - un peu de farine, des vêtements, voire du bétail. Les critères relèvent à la fois de l'examen du niveau de détresse matérielle et de mérite vis-à-vis du régime. Cette démarche s'inscrit dans la prolongation des logiques de distribution de l'entre-deux-guerres, à la nouveauté près que le critère du mérite est désormais fortement corrélé avec l'expérience de la guerre.

Les familles de combattants sont, en principe, prioritaires. Elles relèvent d'une administration spécifique, mise en place pendant la guerre. Au niveau local, le département pour l'aide sociale d'État et l'installation des familles de soldats (otdel po gosobespečeniju i bytovomu ustrojstvu semej voennoslužaščih) est chargé de verser des pensions spécifiques pour certaines familles de combattants et de prendre un certain nombre de mesures plus ponctuelles. On ne dispose pas du nombre exact de récipiendaires pour le district de Slancy même si l'on sait que le pourcentage de familles de combattants est important dans l'oblast de Leningrad et en général en URSS. Cependant, toutes les familles de combattants ne bénéficient pas d'une aide sociale spécifique. Ainsi, au début de l'année 1945, l'oblast de Leningrad comptait 79800 familles de combattants, dont seulement 22230 touchaient des allocations régulières et 8900 percevaient une pension pour la perte du chef de foyer. Pourtant, bien des familles qui ne correspondaient pas aux critères d'une aide sociale fixe de par leur composition, faisaient l'objet d'une aide sociale ponctuelle. Le responsable du département en charge des familles combattantes de la région estimait que 15000 d'entre elles manquaient de nourriture, 36000 , soit près de la moitié, manquaient de vêtements et de chaussures ${ }^{81}$. À Slancy comme dans le reste de l'Union soviétique, les accusations de malversations et d'incurie à l'égard de ce secteur allaient bon train ${ }^{82}$.

81. CGA SPb, f. 7179, op. 19, d. 26, 1. 42-42 ob, cité in Iz rajonov oblasti.., p. 544-546. L'oblast de Leningrad comptait pour la même période une population totale de 474233 personnes, ibid, p. 547, ce qui donne un nombre approximatif de 135495 familles (en appliquant un coefficient de 3,5 personnes par famille). Ainsi plus de la moitié des familles de l'oblast, $59 \%$, seraient des familles de combattants en ces derniers mois de guerre. En appliquant les mêmes critères à la population de Slancy, on obtient environ 990 familles de combattants.

82. Beate Fizeler [Beate Fieseler], « "Niščie pobediteli” : invalidy Velikoj Otečestvennoj vojny v Sovetskom Sojuze » [Les pauvres vainqueurs : Les invalides de la Grande Guerre patriotique en Union soviétique], in Gabovič, réd., Pamiat' o vojne 60 let spustia..., p. 577-591. Cf. aussi Marc Edele, «Soviet Veterans as an Entitlement Group, 1945-1955 », Slavic Review, 65 (1), 2006, p. 111-137. 
Si les familles de frontoviki reviennent comme un leitmotiv parmi les foyers prioritaires de l'aide sociale, d'autres catégories, qui les recoupent parfois, s'y agrègent : les soldats eux-mêmes, en tant qu'invalides ou démobilisés, mais aussi les rapatriés et les personnes de retour d'évacuation. Parmi ces derniers, les plus pauvres reçoivent une aide ponctuelle, au gré des arrivages en provenance de l'exécutif de la région.

Les partisans bénéficient également d'un statut officiel, aussi bien dans la délivrance de pensions que dans la distribution de l'aide sociale. En revanche, le fait d'avoir aidé les partisans ne procure pas un statut en tant que tel. Néanmoins, comme nous l'avons vu, il avait donné lieu à l'élaboration d'une liste d'habitants à privilégier. Des villages entiers sont également distingués comme ayant fait preuve d'un héroïsme particulier pendant la guerre. Au début de l'année 1945, dans leur demande d'aide adressée à Leningrad, les responsables du district de Slancy signalent ainsi 13 villages pour leur conduite pendant la guerre ${ }^{83}$. En revanche, le président du sel'sovet d'un district voisin peine à nommer des localités héroïques puisqu'aucun village du district n'a passé l'ensemble de l'occupation caché dans les bois, seulement quelques-uns au début de la guerre, en juin-juillet 1941, ou dans les trois derniers mois de 1943, au moment du départ des Allemands ${ }^{84}$ : ces moments de calvaire ne correspondaient apparemment pas à la définition de villages résistants aux yeux de ce responsable local.

En raison du niveau de misère de la population et de la rareté des biens à distribuer, la désignation des bénéficiaires est forcément un casse-tête dans lequel entre une bonne part d'arbitraire. Les responsables, s'ils peuvent en tirer quelque avantage personnel, le plus fréquemment, sont accusés d'incompétence ou de prévarication. Ce qu'illustre la distribution d'un bien d'une valeur exceptionnelle - les têtes de bétail —, qualifié de « trophée » car récupéré sur les Allemands dans les fermes baltes. Une première liste nominative est établie en juillet 1945 par F.A. Sedakov, responsable du département pour l'aide sociale d'État, et ratifiée début août, afin de répartir les 395 têtes de bétail entre des foyers de frontoviki, d'invalides de guerre et de partisans qui ne possèdent pas de bétail en bien personnel ${ }^{85} .328$ bêtes sont destinées à des familles de soldats, 32 à des invalides de guerre, 13 à des familles de partisans, 22 enfin sont destinées aux exploitations agricoles d'entreprises et d'administration du district. Début septembre, la liste est modifiée : 181 noms de la première liste sont conservés mais 63 en ont été supprimés, soit que les familles en question aient été fichées pour s'être compromises durant l'occupation (kompromaterial), soit qu'elles n'aient pas correspondu aux critères, ne comptant pas de

83. Lugov'e, Zasjast’e, B. et M. Rožki, Ryžikovo, Dvorišče, Pačtilejkovo, M. Gajansščina, Novinka, Pesevicy, Klin, Gorbovo, Iščevo, Vzjazyšče. LOGAV, f. 624, op. 1, d. 58, 1. 1-3.

84. LOGAV,f. 2019, op. 1, d. 31.

85. En février 1945, le responsable du département pour l'aide sociale d'État v/sl au niveau de la région de Leningrad plaidait pour que 10000 vaches «trophées » soient distribuées aux familles de frontoviki les plus nécessiteuses, seule manière à ses yeux de véritablement améliorer leur sort. CGA SPb, f. 7179, op. 19, d. 26, 1. 42-42 ob, cité in Iz rajonov oblasti..., p. 544-546. 
soldat, ou possédant déjà leur propre vache. En revanche, 12 familles de retour d'évacuation ont été rajoutées, prouvant l'émergence d'une nouvelle catégorie d'indigents prioritaires. À la mi-septembre, Sedakov est limogé de son poste. La constitution de ces listes erronées est un des principaux reproches qui lui sont adressés ${ }^{86}$.

Comme nous l'avons déjà souligné, les mauvaises méthodes de Sedakov s'expliquent en partie par l'importance des connaissances personnelles dans une communauté de taille réduite, ce à quoi s'ajoute le fonctionnement de l'aide, qui repose pour beaucoup sur l'observation des conditions de vie des familles comme critère pour bénéficier de telle ou telle aide. Le fait d'avoir insuffisamment prêté attention aux cas concrets, ou encore d'avoir mis sur les listes des personnes qui n'étaient pas parmi les plus nécessiteuses est ainsi fréquemment reproché aux responsables de l'aide sociale ${ }^{87}$.

\section{Aide aux sinistrés et exemptions fiscales}

Le fardeau de l'impôt et des livraisons obligatoires qui pèsent lourdement sur la paysannerie soviétique au sortir de la guerre a été bien mis en évidence par les historiens. Ven'iamin Zima a ainsi montré comment ce fardeau explique l'ampleur de la famine de 1946, la sécheresse apportant le coup de grâce à une paysannerie particulièrement frappée par la guerre et dont l'État, au lieu de l'aider à se relever, ponctionne la production. Les figures des agents fiscaux et des plénipotentiaires du commissariat aux livraisons venant prélever, de gré ou de force, leur dîme ont également été évoquées par V. Popov, qui souligne la part importante des foyers paysans tout simplement incapables de s'acquitter des sommes et des produits exigés par la puissance publique. Cependant les anciens territoires occupés ne sont jamais considérés comme des objets d'études particuliers par ces historiens ${ }^{88}$.

Nous voudrions donc examiner ici un aspect mal connu, celui des exemptions fiscales, partielles ou totales, accordées à certains foyers, au sein d'une population effectivement pressurée par divers impôts et livraisons obligatoires. Les bénéficiaires relevaient de catégories définies, à la fois sur des critères « statutaires », notamment certaines familles de combattants, en fonction du ratio entre adultes valides/enfants et vieillards à charge, et sur des critères matériels, faisant intervenir la notion de foyers «particulièrement victimes de l'occupation », c'est-à-dire sinistrés. L'absence de définition précise rendit nécessaire l'intervention des autorités locales pour désigner les ayants droit. Ainsi, alors que le travail de l'agent des impôts consiste à évaluer pour chaque foyer le montant de l'imposition, l'administration des sel'sovety (en

86. LOGAV, f. 624 , op. 1, d. $47,1.52,60,99,110$.

87. Par ex. LOGAV, f. 624 , op. 1, d. $45,1.3$.

88. V.P. Popov, Ekonomičeskaja politika sovetskogo gosudarstva, 1946-1953 [Politique économique de l'État soviétique, 1946-1953], Tambov, 2000 ;Zima, Golod v SSSR 1946-1947 godov... ; Franklyn D. Holzman, Soviet Taxation: The Fiscal and monetary problems of a Planned Economy, Cambridge : Harvard University Press, 1957. 
réalité une poignée de personnes) dresse des listes de foyers à exempter, examinées et ratifiées ensuite par le comité exécutif du district. Nous retrouvons ici les fonctions pré-modernes de la communauté locale, dans la gestion de l'impôt. Ces mesures d'exemption ne pouvant en aucun cas couvrir l'ensemble des sinistrés, les autorités locales piochent parmi ces derniers, selon des critères qui échappent en grande partie à l'analyse des archives : comment établir une échelle de la détresse matérielle parmi une population constituée en quasi-totalité de sinistrés ?

Ces mesures en matière d'exemptions fiscales sont caractéristiques de l'attitude de l'État soviétique à l'égard des sinistrés. Alors que le travail de la Commission d'enquête soviétique tendait à l'exhaustivité et fit naître, chez certains, l'espoir d'être indemnisés, au moins en partie, telle n'était pas l'intention de l'État soviétique. Il aurait été politiquement et financièrement très difficile d'indemniser les propriétaires sinistrés à la hauteur de leur perte. La reconnaissance des pertes subies par les citoyens soviétiques servait d'autres objectifs, relevant avant tout de la politique étrangère ${ }^{89}$. Pour faire face aux problèmes des sinistrés, l'État soviétique recourt à de vieilles recettes, utilisées dans les cas de catastrophes naturelles et circonstances exceptionnelles, notamment en cas de sécheresse et de récoltes particulièrement désastreuses. Il s'agit donc de mesures purement ponctuelles, même si elles peuvent être reconduites dans le temps, et elles ne concernent que certaines régions. L'autre caractéristique de ces mesures tient au rôle joué par les autorités locales dans la désignation des bénéficiaires. Dans les listes d'habitants sinistrés dressées par l'administration des sel'sovety, une colonne est consacrée à la présence de soldats ou de partisans au sein du foyer. Ces listes ont été utilisées, entre autres usages, pour désigner les foyers exemptés, totalement ou partiellement, du paiement de l'impôt agricole ( sel’hozjajstvennyj nalog). Or, nous constatons que des foyers de sinistrés ne comptant aucun membre dans l'Armée rouge sont exemptés, alors que d'autres, pouvant se prévaloir de morts sur le champ de bataille, ne bénéficient pas de cette disposition ${ }^{90}$.

D'autres listes confirment que les familles de combattants sont loin d'être les seules bénéficiaires de l'exemption d'impôt. Ainsi, en avril 1945, au moment où les territoires libérés sont à nouveau soumis aux livraisons en produits d'origine animale, des kolkhoziens considérés comme particulièrement victimes de l'occupation et dépourvus de bétail ont été exemptés par le district de livraisons obligatoires en viande et en œufs ${ }^{91}$. Sur les 167 familles de la liste des foyers exemptés,

89. Moine, « La Commission d'enquête soviétique... ».

90. LOGAV, f. 629, op. 1, d. 17, spisok postradavščih hozjajstv po Savinovščinskomu s/sovetu na $1945 \mathrm{~g}$. Slancevskogo r-na [liste des foyers sinistrés établie pour 1945 pour le sel'sovet de Savinovščin, district de Slancy].

91. Postanovlenie SNK SSSR n 670, 4 avril 1945, Popov, Ekonomičeskaja politika... p. 193. Le décret s'appliquait également aux livraisons de la part des kolkhozes. Lors de la discussion préliminaire sur les termes exacts du décret, le commissariat à l'industrie carnée et laitière avait fait remarquer qu'au vu du grand nombre de kolkhozes dépourvus de tout bétail dans les régions libérées (près d'un sur 5 dans l'oblast de Leningrad), le nombre d'impayés risquait d'être conséquent et la mesure prématurée. Il préconisait donc un autre mode de calcul, basé sur le nombre effectif de têtes de bétail. Popov, Krest'janstvo i gosudarstvo (1945-1953) [Les paysans et l'État (1945-1953)], P. : YMCA-Press, 1992, p. 187-189. 
140 ne comptent pas de soldat. Une seule a vu un des siens tué dans les rangs des partisans ${ }^{92}$. Rappelons que ces livraisons sont exigées aussi bien de foyers sans bétail $^{93}$. L'exonération ne constitue en rien une mesure exhaustive, le nombre de foyers dépourvus de bétail étant bien supérieur à celui des exemptés : dans une note de février 1945 adressée au comité exécutif de la région, Ivanov indiquait que pas moins de 598 foyers de kolkhoziens sur 1055 étaient dépourvus de bétail ${ }^{94}$. Ainsi, le nombre de foyers du district auxquels on soutira œufs et viande, alors qu'ils ne possédaient aucune tête de bétail, fut très certainement bien supérieur au nombre d'exemptés.

Parmi les personnes inscrites sur cette liste, nous trouvons Varvara Ivanovna Germanovna, par ailleurs présidente de la commission de révision du kolkhoze «Udarnik » (présidé par M.P. Germanov), ou encore Marija Kont, membre de la commission de révision du kolkhoze «3ij Reščajuščij». Situation d'extrême pauvreté et favoritisme semblent ainsi inextricablement mêlés : si nous pouvons soupçonner que la parcelle de pouvoir détenue par ces femmes les a aidées à bénéficier de cette aide, elles n'en partagent pas moins la misère commune à l'ensemble des habitants. Nous trouvons également une certaine Anna Kondrat'evna Martynova, du village de Gosticy, membre du kolkhoze « Den' urožaja », à la tête d'une famille de 5 personnes dont trois adultes valides, et qui compte parmi les familles de frontoviki. Quelques mois plus tard, elle obtient d'utiliser une construction laissée par l'Armée rouge pour se reconstituer un toit. Le cas de la famille Martynov montre la corrélation entre la tenue d'une charge officielle et l'accès à des mesures d'aide, y compris dans des familles traversées par la ligne de fracture entre anciens collaborateurs et résistants. La présence d'un Ja.T. Martynov à la direction du kolkhoze «Den' urožaja » n'est pas incompatible avec le niveau de pauvreté de la famille mais tend à prouver qu'elle possède cette précieuse once de pouvoir local. Et ce, en dépit de l'inscription en février 1944 d'un autre Martynov originaire du même village (sans que l'on puisse établir le degré de parenté), sur une liste de policiers enfuis avec les Allemands ${ }^{95}$.

92. LOGAV, f. 624, op. 1, d. 45, 1. 112 ; f. 624, op. 1, d. 45, 1. 113-115 ob, spisok hozjajstv kolhoznikov Slancevskogo rajona osobenno postradavših ot nemeckoj okkupacii i neimejuščih v ličnom pol'zovanii skota [Liste de foyers de kolkhoziens du rajon de Slancy ayant particulièrement souffert de l'occupation allemande et ne disposant pas de bétail pour leur usage personnel].

93. Popov, Ekonomičeskaja politika... p. 186. Bien souvent, les collecteurs acceptaient des produits de substitution, ou l'équivalent en numéraire, selon les prix du marché kolkhozien. En 1950, pour toute l'URSS, 16 millions de vaches appartenaient à des particuliers, mais 18 millions de foyers étaient censés se plier à des livraisons de viande et lait, soit au moins 2 millions de foyers sans bétail. Ibid, p. 192.

94. LOGAV, f. 624, op. 1, d. 58,1.1-3.

95. LOGAV, f. 624 , op. 1, d.45, 1.114 ; d. 47, 1.64 (A.K. Martynova) ; d. 13, 1.39 (Ja.T. Martynov) ; Musée de Slancy, Spisok policejskih i lic, sostojavših v kar. Otr. [Liste des policiers et personnes appartenant au détachement punitif]. Cf. aussi Antonina Trifonovna Martynova, du même sel'sovet, exemptée à $100 \%$ du paiement de l'impôt agraire (sel’hoznalog) en décembre 1945, GARF, f. A-259, op. 6, d. 3761, 1. 12. Cf. aussi le cas noté plus haut de la famille Burmistrova, note 74. 


\section{Reconstruire son toit dans le district de Slancy}

En raison des aléas du front, ce n'est qu'à la fin de l'année 1944 que les villages du district commencent à être, très progressivement, relevés. Or, les autorités comme les habitants eux-mêmes se heurtent à un manque cruel de matériaux de construction. Aux dires d'Ivanov à la fin de l'année 1944, sur 1640 foyers, 1 265, soit 77 \%, vivent encore dans des constructions temporaires..$^{96}$

Dans une note d'information de 1945, malheureusement sans indication du mois, nous trouvons la répartition suivante : la moitié des kolkhoziens sont encore privés de maisons, soit 616 foyers, mais la proportion s'élève à $70 \%$ chez les paysans récalcitrants au collectivisme (le district compte encore 230 foyers d'exploitations individuelles d'edinoličniki $\left.i^{97}\right)$. Cette proportion est à peu près similaire $(65 \%)$ pour les foyers d'ouvriers et d'employés vivant dans un village (310 au total) ou bien dans le bourg mais disposant d'un terrain (281 au total) ${ }^{98}$. En octobre 1945, le comité exécutif dresse une liste de 135 foyers, soit 430 personnes, vivant encore dans des zemljanki ${ }^{99}$. À l'automne 1945, la situation est jugée particulièrement préoccupante pour les kolkhoziens des collectivités les plus frappées par les destructions (sel'sovety Vyskatskij, Savinovščinskij, Popkovo-Gorskij et Pelešskij). 37 familles habitent encore dans des zemljanki dans le sel'sovet Vyskatskij, 15 dans le Savinovšinskij, 16 dans le Popkovo-Gorskij, 18 dans le Pelešskij. Les présidents des sel'sovety constituent des boucs émissaires tout trouvés pour ces carences, et l'un d'entre eux, Belov, président du sel'sovet Vyskatskij, sera limogé peu de temps plus tard, au début de l'année 1946, en partie sur ce motif ${ }^{100}$.

Pour faire face à ce défi, les méthodes habituelles de mobilisation sont utilisées : des journées sont, par exemple, consacrées à l'aide aux familles de soldats particulièrement nécessiteuses pour les assister dans la reconstruction de leurs maisons. Sedakov est responsable de l'opération « décade d'aide aux familles de soldats au front » (dekadnik pomošči sem jam frontovikov) destinée aux foyers de combattants dépourvus d'adultes valides et aux femmes de soldats, mères de familles nombreuses; elle se tient du 27 octobre au 5 novembre 1944 et, au cours de cette dizaine de jours, les membres du komsomol sont censés prêter main forte pour restaurer et reconstruire les maisons. Mais là encore cette aide est partielle et ne concerne qu'un nombre limité de personnes, enregistrées sur une liste de familles de frontoviki particulièrement nécessiteuses ${ }^{101}$.

96. CGA SPb, f. 7179, op. 14, d. 26,1. 176-177 ob. Cité in Iz rajonov oblasti..., p. 525.

97. Jean Lévesque, «"Into the grey zone”. Sham peasants and the limits of the kolkhoz order in the post-war Russian village, 1945-1953 », in J. Fürst, éd., Late Stalinist Russia: Society Between Reconstruction and Reinvention, London : Routledge, 2006, p. 103-119.

98. LOGAV, f. 624, op. 1, d. 58,1. 131.

99. LOGAV, f. 624, op. 1, d. 58, 1. 180-183, Spisok kolhoznikov proživajuščih v zemljankah po Slancevskomu rajonu po sostojaniju na 10 oktjabrja $1945 \mathrm{~g}$. [Liste des kolkhoziens vivant dans des constructions en terre dans le rajon de Slancy, établie au 10 octobre 1945].

100. LOGAV, f. 624, op. 1, d. 47,1. 142.

101. LOGAV, f. 624, op. 1, d. 13,1.79. 
La reconstruction du bourg de Slancy lui-même rencontre des difficultés analogues, même si, en définitive, beaucoup plus de moyens seront mis en œuvre, grâce à la présence d'entreprises épaulées par Leningrad. Ivanov demande à plusieurs reprises de disposer de matériel de construction. Ses requêtes concernent aussi le personnel : il souhaite maintenir dans son district les quelques personnes qualifiées en matière de construction, et de bénéficier d'une main d'œuvre supplémentaire, extérieure au district, ainsi que des prisonniers de guerre du camp 322.

$\mathrm{Au}$ sein des kolkhozes se constituent des brigades de construction qui font l'objet de tensions perpétuelles en raison du manque cruel de main-d'œuvre. Pour la même raison, de fortes tensions se font sentir entre les kolkhozes et les entreprises de Slancy, qui attirent à elle des kolkhoziens et une partie des réévacués, sans pour autant avoir sollicité les autorisations du comité exécutif, des directions de kolkhozes et des sel'sovety ${ }^{102}$.

L'un des grands problèmes auquel se trouvent confrontés tous ceux, particuliers ou kolkhoziens, qui entreprennent la reconstruction des maisons est le manque de bois. Or, les constructions rudimentaires, domiki, abandonnées par la troupe, et les stocks de bois sont soumis à une surveillance drastique, leur distribution fait l'objet, là encore, d'une décision nominative du comité exécutif et apparaît ainsi comme une faveur légitimée par plusieurs facteurs. En octobre 1944, 40 domiki sont données au sel'sovet Pelšinskij, 50 au Vyskatskij, afin de couvrir les besoins les plus urgents des kolkhoziens. Cependant, toute construction laissée par l'armée, si elle peut être utilisée à des usages civils, doit recevoir l'autorisation du comité exécutif et fait l'objet d'un paiement à l'administration forestière du district ${ }^{103}$. En octobre 1945, conformément à la législation prise en faveur des démobilisés de l'Armée rouge, il est à nouveau fait mention d'une distribution, exclusivement sur liste nominative, des constructions militaires de 1944, ainsi que du bois stocké par le gosfond ${ }^{104}$. Le tout est distribué en priorité aux habitants démobilisés des poselki de Slancy.

La tâche de reconstruction des maisons bénéficie également d'une nouvelle procédure, à savoir l'allocation d'un crédit individuel, au montant variable mais qui ne pouvait dépasser 10000 roubles, assorti, en principe, d'un accès prioritaire aux matériaux de construction ${ }^{105}$.

102. LOGAV, f. 624, op. 1 ; d. 13,1.92; f. 624, op. 1, d. 47,1. 45.

103. LOGAV, f. 624 , op. 1 , d. $13,1.111$.

104. LOGAV, f. 624 , op. 1, d. $47,1.45$, etc.

105. Un premier décret portant sur le crédit à la construction individuelle est étendu aux paysans à la faveur de la loi sur les démobilisés du 23 juin 1945. Postanovlenie SNK SSSR, 29 mai 1944, «o meroprijatijah po vosstanovleniju individual'nogo žiliščnogo fonda v osvoboždennyh rajonah i usileniju individual'nogo žiliščnogo stroitel'stva v gorodah i rab. poselkah SSSR », rešenija partii i pravitel'stva po hozjajstvennym voprosam, vol. 3, M., 1968, p. 205-7 [Arrêté SNK SSSR du 29 mai 1944 sur « les mesures pour rétablir le fonds d'habitat individuel dans les districts libérés et renforcer la construction d'habitat individuel dans les villes et les villages ouvriers d'URSS », décisions du parti et du gouvernement aux questions économiques]. Mark Edele, «A "Generation of Victors ?" Soviet Second World War Veterans from Demobilization to Organization, 1941-1956», Phd dissertation, University of Chicago, 2004, p. 125. 
Là encore, si les responsables du district font partie des bénéficiaires, c'est parce qu'ils appartiennent à des catégories de victimes prioritaires : c'est le cas de Dar'ja Petuhova, à qui, en tant que parente de frontovik, est accordé un crédit de 4000 roubles en juillet 1945 ; Sedjakov perçoit 8000 roubles de crédit en août 1945 , peu avant son limogeage; Fedor Iakovlevich Krjukov, président du sel'sovet de Popko-Gorskij, a droit, en tant que démobilisé, à plusieurs mètres cubes de bois ainsi qu'à un crédit de 6000 roubles, tout en étant blâmé pour la lenteur de la reconstruction des maisons des kolkhoziens. Georgij Semenovič Semenov, lui aussi démobilisé, reçoit 10000 roubles le jour de la confirmation de son élection comme président du comité exécutif du sel'sovet Novosel'skij; en janvier 1946, Mihail Alekseevič Gubernatorov, 3e secrétaire du comité du Parti, en tant que démobilisé et ancien partisan, bénéficie d'un crédit de 5000 roubles ; quelques mois plus tard, à l'automne 1946, Marija Sergeeva, travaillant dans une usine avant-guerre, membre de la résistance clandestine du district pendant l'occupation, devenue fonctionnaire du comité du parti du district, se voit octroyer la somme maximale de 10000 roubles $^{106}$. En revanche, nous ne savons pas ce qui vaut à une certaine Lidija Fedorovna Pokin'čereda, revenue de réévacuation (ou rapatriée) dans le bourg de Slancy, de profiter de deux crédits, l'un de 5000 roubles à la fin de 1945, l'autre de 10000 roubles en 1946, puis de recevoir un petit pécule de 200 roubles destinés aux plus nécessiteux des rapatriés et réévacués. Faisant partie des familles de frontoviki, la kolkhozienne Ekaterina Egorovna Kuz'mina, du kolkhoze « Šahter », exemptée de livraisons faute de bétail, reçoit en outre 4000 roubles en crédit pour reconstruire son toit ${ }^{107}$.

Le fait d'avoir accès à ces crédits facilitait-il vraiment la reconstruction d'une maison, sachant que le problème crucial était d'avoir accès aux matériaux euxmêmes ? Et dans quelle mesure l'argent était-il utilisé à d'autres fins que la reconstruction elle-même ? Ainsi, le citoyen Tarnovskij, qui aurait utilisé $10 \%$ du crédit reçu (ssuda) à d'autres usages, se voit retirer la somme, avec une majoration de $12 \%$, au printemps $1946^{108}$. Enfin, dans quelle mesure les bénéficiaires du prêt purent-ils s'acquitter de leur dette ?109

À l'inverse, qui reste vivre dans les zemljanki ? Les personnes les plus discréditées en raison de leur conduite pendant la guerre ? Les kolkhoziens dépourvus de toute influence ou incapables, physiquement et matériellement, de reconstruire leur maison? Le niveau de dévastation subie par le village entre également en compte.

106. LOGAV, f. 624, op. 1, d. 47, 1.55 (Petuhova), 1. 64 (Sedjakov), 1.74 et 81 (Krjukov), d. 70,1.33 (Semenov), 1. 66 (Gubernatorov), d. 71,1.141 et Musée de Slancy, « spisok aktivno rabotajuščih v podpol’nyh organizacii Slancevskogo rajona » et «spisok lic okazyvajuščie pomošči partizanam s 16.07.41g. po 1.02.44g. », (Sergeeva).

107. LOGAV,f. 624, op. 1, d. 70,1. 65-66 et 164 (Pokin'́̌ereda), 1. 275 (Kuz'mina).

108. LOGAV, f. 624, op. 1, d. 70,1. 213. Cf. aussi f. 624, op. 1, d. 70,1. 105.

109. Cf. la demande du secrétaire du parti d'une autre région ayant subi l'occupation, l'oblast de Smolensk, adressée à Molotov le 29 juin 1953 pour demander, entre autres, l'extinction de la dette contractée par les kolkhoziens auprès de la sel'hozbank pour construire leur maison. GARF, f. 5446, op. 87, d. 1058,1. 21-23, cité in Popov, Krest’janstvo..., p. 220-222. 
Dans le kolkhoze « Rožki », où 37 maisons sur 38 ont été anéanties, et qui, au dire d'Ivanov fait partie des villages au comportement exemplaire pendant la guerre ${ }^{110}$, le président du kolkhoze lui-même, son comptable et 5 autres kolkhoziens vivent toujours dans des huttes à la fin de 1945. On trouve aussi dans les zemljanki des familles pourtant considérées comme plus victimes que les autres dans la hiérarchie officielle et donc en principe prioritaire: ainsi la famille de la citoyenne E.D. Fomina, à laquelle on enlève ses deux enfants en janvier 1946, Valentina née en 1937 et Anatolij né en 1939, pour les placer dans un orphelinat. La femme souffre de troubles mentaux, ils vivent dans une zemljanka dans le village de Gosticy, sans vêtements, sans nourriture. Or, le père a été fusillé par les Allemands en $1944^{111}$.

Malgré le caractère relativement massif de l'obtention de prêts à la construction qui, d'après les listes du comité exécutif chargé de les octroyer, concerne environ 180 familles au cours des années 1945-1946, la mesure reste discrétionnaire, et il est évident que tous ceux qui reconstruisirent leur maison ne purent bénéficier des prêts. Indépendamment ou non de cette faveur, l'ensemble des bâtisseurs étaient censés respecter un cahier des charges, portant tant sur la maison elle-même que sur son emplacement. Ces instructions furent loin d'être toujours respectées, malgré la menace de démolition qui pesait sur les contrevenants, de sorte que les autorités, ici comme dans d'autres régions de l'URSS, se plaignirent du caractère anarchique des nouveaux bâtis ${ }^{112}$.

Le bourg de Slancy fit l'objet de plus d'attention dans la planification de sa reconstruction, même si cette supériorité est toute relative. La présence de grosses entreprises comme le combinat Leningradslanec, les entreprises «Lengazprovodstroj » et «Giprošahta » donnent du poids à la nécessité de reloger leurs personnels et de leur procurer des équipements publics, puisque ce sont les entreprises elles-mêmes qui prennent en charge logement et entretien. En février 1946, le comité exécutif étudie des projets de développement pour Slancy et les villages de B. Lučki et Zamoš́e. Il est prévu que Slancy accueillera une population de 15000 personnes, incluant les travailleurs de la mine Kirov, de la station électrique (CES), d'une briquetterie et d'autres usines, alors que la population de la ville nouvelle projetée à B. Lučki devrait atteindre 22000 personnes, comprenant les travailleurs des mines 1, 2, 3, 4, ceux de l'usine de gaz de Slancy, d'une briquetterie et d'autres entreprises ${ }^{113}$. Afin de surveiller la mise en œuvre du projet, un architecte de la ville est nommé, l'ingénieur urbaniste Mug. À partir d'octobre 1946, Aleksej Semenovič Efimov, géomètre, officie comme ingénieur urbaniste de la ville de Slancy ${ }^{114}$.

Les plans comprennent une ceinture forestière de $5 \mathrm{~km}$, conformes en cela à l'urbanisme soviétique. D'autres aspects sont caractéristiques du mode de vie

110. Cf. note 84 .

111. LOGAV, f. 624, op. 1, d. 70,1. 34.

112. LOGAV, f. 624, op. 1, d. 70,1. 2 (janvier 1946).

113. LOGAV, f. 624, op. 1, d. 70, 1. 57.

114. LOGAV, f. 624, op. 1, d. 71, 1. 183. 
d'une bourgade industrielle dont les habitants ont non seulement conservé des habitudes rurales mais sont invités à les maintenir : pâturage, potagers et pépinières. Bien entendu, d'après les rapports sur l'état sanitaire de Slancy, nous sommes loin, dans les faits, d'une cité modèle ${ }^{115}$.

\section{Vers la famine de 1946}

Alors que les villageois sortent progressivement de leur logement de fortune pour retrouver de vraies maisons même si, bien souvent, elles sont faites de bric et de broc, les autorités bataillent pour revenir à un niveau décent dans la distribution «normale» de biens de première nécessité : produits alimentaires, mais aussi produits manufacturés de base comme les vêtements, les chaussures, le mobilier, le savon, les allumettes... Elles tentent de remettre sur pied une production locale mais se heurtent aux insurmontables contraintes en main-d'œuvre et matières premières. Le limogeage de Barinov, directeur de l'entreprise de produits manufacturés du district, au printemps 1946, reflète moins sa propre incurie que les problèmes chroniques rencontrés par la production de chaussures ou de vêtements, faute de salaires et de conditions de vie décente des employés ${ }^{116}$. Ivanov envisage même d'utiliser le travail des prisonniers de guerre cantonnés à proximité dans le camp $n^{\circ} 322$ pour la fabrication de jouets ${ }^{117}$.

Ainsi, l'offre reste très inférieure à la demande, aussi bien en produits manufacturés de base qu'en produits alimentaires. Le marché kolkhozien de Slancy illustre l'incapacité des organisations publiques à fournir la population. Les deux comptoirs de la coopérative de consommation du district (Rajpotrebsojuz) et de l'entreprise locale de l'industrie alimentaire (Rajpiščekombinat) ne sont pas toujours ouverts. Les autorités se plaignent des visées spéculatives d'acheteurs venant par le train de Leningrad pour acquérir de grosses quantités de produits agricoles qui font grimper les prix ${ }^{118}$.

Aide ponctuelle et distribution fermée restent donc les maîtres mots du fonctionnement de la consommation. Les listes des personnes réévacuées et rapatriées de retour dans les différents sel'sovety, notamment ceux qui font l'objet d'une aide ponctuelle, sont régulièrement révisées ${ }^{119}$. Le nombre des aides distribuées à des personnes de retour d'évacuation ou rapatriées ne cesse d'augmenter, tout en restant quantité négligeable dans le flux des retours.

115. LOGAV, f. 624, op. 1, d. 70. Sur l'absence de gestion des déchets organiques dans les villes soviétiques d'après-guerre, $\mathrm{cf}$. D. Filtzer, «Standards of living versus quality of life : struggling with the urban environment in Russia during the early years of post-war reconstruction », in Fürst, éd., Late Stalinist Russia..., p. 81-102.

116. LOGAV, f. 624, op. 1, d. 70, 1. 209.

117. LOGAV, f. 624 , op. 1, d. 70,1.75-76.

118. LOGAV, f. 624 , op. 1, d. 70, 1. 89-90.

119. LOGAV, f. 624 , op. 1, d. $45,1.46$. 
$\mathrm{Au}$ rationnement dont bénéficient les travailleurs de Slancy correspondent simplement des livraisons de grains destinés aux kolkhoziens se trouvant dans les situations de pénurie les plus dramatiques ${ }^{120}$.

D'autre part, les responsables du district continuent à figurer parmi les habitants particulièrement dans le besoin auquel le district distribue des aides en argent et en nature (produits alimentaires, vêtements). C'est le cas d'Aleksej Semenovič Efimov, ingénieur urbaniste de la ville de Slancy qui reçoit une aide de 500 roubles à l'automne $1946^{121}$. Cependant, signe de l'exacerbation de la pénurie, des rations alimentaires spécifiques sont assignées aux enfants et personnes à charge des « notables » du district. Alors qu'en septembre seize dirigeants figurent sur la liste, leur nombre descend à six quelques jours plus tard. Reflet attendu du sommet de la hiérarchie du district, les rescapés sont A.K. Gončarov, $1^{\mathrm{er}}$ secrétaire du comité du parti au niveau du district, A.D. Malyšev, 2e secrétaire, M.A. Gubernatorov, 3e secrétaire, M.O. Ivanov, président du comité exécutif du soviet du district, G.V. Matorin, son vice président et A.V. Čmutina, secrétaire du comité exécutif. Sont passés par pertes et profits de la disette les directeurs des départements les plus importants du comité exécutif, parmi lesquels le poste de directeur de l'aide sociale ne figure d'ailleurs pas ${ }^{122}$.

\section{Conclusion}

Des contemporains de la Grande Guerre patriotique aux historiens actuels, personne n'a jamais douté de l'importance fondamentale de l'expérience de la guerre dans la reconfiguration des identités des citoyens soviétiques d'aprèsguerre, pour le meilleur et pour le pire. Ce constat apparaît renouvelé dans le cas très concret de la désignation des bénéficiaires de l'aide publique dans un district situé dans les ex-territoires occupés. Il se traduit par l'activité incessante des autorités locales pour dresser des listes de leurs concitoyens nécessiteux qui sont avant tout des familles de frontoviki ou d'anciens partisans. Les démobilisés, les invalides de guerre, les familles de frontoviki et de soldats morts au combat constituent des catégories qui reviennent inlassablement parmi les bénéficiaires officiels. D'autres critères liés à la guerre apparaissent également telle la destruction totale des biens d'un foyer ou la perte de son bétail personnel. Cette situation de sinistrés ne donne pas lieu à l'élaboration d'un véritable statut et ne constitue qu'un critère parmi d'autres dans l'attribution de l'aide. Localement cependant, elle a pu jouer un rôle important dans l'attribution de maigres faveurs.

Au-delà de l'importance du sceau de la guerre, la définition extrêmement floue des personnes susceptibles de bénéficier de l'aide publique, qu'elle soit régulière ou ponctuelle, donne un rôle essentiel aux connaissances interpersonnelles et aux

120. LOGAV, f. 624, op. 1, d. 70,1. 194-195.

121. LOGAV, f. 624, op. 1, d. 71, 1. 183.

122. LOGAV, f. 624 , op. 1 , d. $71,1.125,141$. 
relations de pouvoir, aussi infimes soient-elles. Aux responsables locaux de désigner qui furent les plus grandes victimes de l'occupation, mais aussi qui furent les traîtres, épurés ou relégués hors de la manne publique. Passé sous silence dans les récits officiels, ce travail de tri entre loyaux et déloyaux est mené intensivement par les nouvelles autorités, lors du travail de bilan mené aussi bien par la Commission d'enquête soviétique que par les responsables de la sécurité d'État, dont le travail reste malheureusement inaccessible aux chercheurs d'aujourd'hui.

Cette responsabilité des autorités locales autorise toutes les suspicions, celle de servir en premier lieu ses connaissances, établissant ainsi une véritable clientèle, ou de se servir soi-même, un comportement qui est apparu à plusieurs reprises, à Slancy comme ailleurs. Cependant, cette observation est à double tranchant : elle montre aussi bien le dénuement extrême de tous, y compris des plus puissants, pour lesquels un costume, une paire de chaussures, quelques planches de bois, sans parler d'une vache, constituent une véritable aubaine.

Dès lors, c'est l'ensemble de la communauté qui cherche à se préserver de la pénurie totale. Sans doute, une justice sociale s'exerce localement pour accorder une aide à ceux dont la conduite n'eut rien d'héroïque pendant la guerre mais que les destructions et l'isolement ont réduit à une quasi-mendicité.

Néanmoins, si nous voulons in fine qualifier l'aide matérielle dispensée à la libération, nous constatons son caractère très ponctuel, très limité par rapport au degré de misère et de destruction, et très circonscrit : les exemptions et les aides ne sont reçues que par une fraction des plus démunis désignés en partie sur des critères statutaires qui privilégient les services rendus au pouvoir, alors que le niveau d'imposition, en numéraire comme en nature ne cesse de s'accroître. Les mesures d'assistance à la population de sinistrés apparaissent ainsi très en deçà du défi de la reconstruction. C'est cette population particulièrement fragile qui se retrouve frappée par le fléau de la famine en 1946, rendant définitivement peu adapté le terme de « reconstruction ».

EHESS, Centre d'études des mondes russe, caucasien et centre-européen

Nathalie.Moine@ehess.fr 